AperTO - Archivio Istituzionale Open Access dell'Università di Torino

\title{
Performance and air-shower reconstruction techniques for the JEM-EUSO mission
}

\section{This is a pre print version of the following article:}

Original Citation:

Availability:

This version is available http://hdl.handle.net/2318/156048

since

Published version:

DOI:10.1016/j.asr.2014.02.018

Terms of use:

Open Access

Anyone can freely access the full text of works made available as "Open Access". Works made available under a Creative Commons license can be used according to the terms and conditions of said license. Use of all other works requires consent of the right holder (author or publisher) if not exempted from copyright protection by the applicable law. 


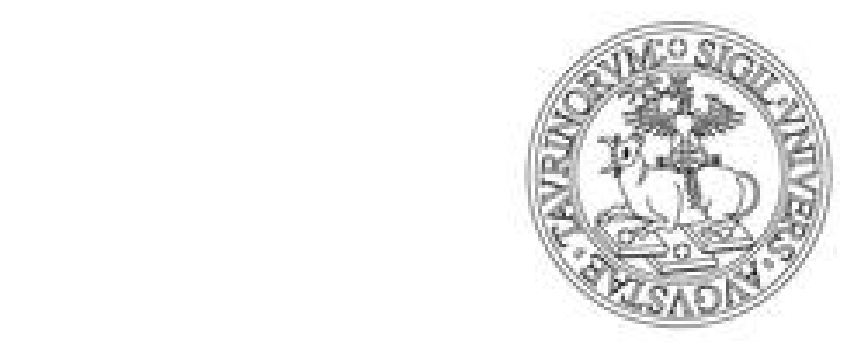

\section{UNIVERSITÀ DEGLI STUDI DI TORINO}

This Accepted Author Manuscript (AAM) is copyrighted and published by Elsevier. It is posted here by agreement between Elsevier and the University of Turin. Changes resulting from the publishing process - such as editing, corrections, structural formatting, and other quality control mechanisms - may not be reflected in this version of the text. The definitive version of the text was subsequently published in ADVANCES IN SPACE RESEARCH, 53, 2014, 10.1016/j.asr.2014.02.018.

You may download, copy and otherwise use the AAM for non-commercial purposes provided that your license is limited by the following restrictions:

(1) You may use this AAM for non-commercial purposes only under the terms of the CC-BY-NC-ND license.

(2) The integrity of the work and identification of the author, copyright owner, and publisher must be preserved in any copy.

(3) You must attribute this AAM in the following format: Creative Commons BY-NC-ND license (http://creativecommons.org/licenses/by-nc-nd/4.0/deed.en), 10.1016/j.asr.2014.02.018

The definitive version is available at:

http://dx.doi.org/10.1016/j.asr.2014.02.018 
truefalse

$, \quad, \quad, \quad, \quad,,,,,,,,,,,$, 


\title{
Performance and air-shower reconstruction techniques for the JEM-EUSO mission
}

\author{
M. Bertaina ${ }^{\mathrm{a}, \mathrm{b}, *}$, S. Biktemerovac ${ }^{\mathrm{c}}$, K. Bittermann ${ }^{\mathrm{d}, 1}$, P. Bobik ${ }^{\mathrm{e}, *}$, \\ D. Campana ${ }^{\mathrm{f}}$, F. Fenu ${ }^{\mathrm{d}, *}$, A. Gorgi ${ }^{\mathrm{a}, \mathrm{b}}$, F. Guarino ${ }^{\mathrm{f}, \mathrm{g}}$, A. Guzmán ${ }^{\mathrm{d}, *}$, \\ K. Higashide ${ }^{h, i}$, G. Medina-Tanco ${ }^{\mathrm{j}}$, T. Mernik ${ }^{\mathrm{d}, *}$, D. Naumov ${ }^{\mathrm{c}}$, M. Putis $^{\mathrm{e}}$, \\ M.D. Rodríguez Frías ${ }^{k}$, G. Sáez Cano ${ }^{k}$, A. Santangelo ${ }^{d}$, K. Shinozaki ${ }^{\text {,i,*, }}$, \\ and S. Toscano ${ }^{1}$, for the JEM-EUSO Collaboration ${ }^{m}$ \\ ${ }^{a}$ Dipartimento di Fisica, Università di Torino, Via Giuria 1, I-10125, Torino, Italy \\ ${ }^{b}$ Istituto Nazionale di Fisica Nucleare - Sezione di Torino, Via Giuria 1, I-10125, \\ Torino, Italy \\ ${ }^{c}$ Joint Institute for Nuclear Research, Joliot-Curie 6, 141980 Dubna, Russia \\ ${ }^{d}$ Institute for Astronomy and Astrophysics, University of Tübingen, Sand 1, D-72076 \\ Tübingen, Germany \\ ${ }^{e}$ Institute of Experimental Physics, Slovak Academy of Sciences, Watsonova 47, \\ SK-04001 Kosice, Slovakia \\ ${ }^{f}$ Istituto Nazionale di Fisica Nucleare - Sezione di Napoli, Complesso Universitario di \\ Monte Sant'Angelo, via Cintia, I-80126, Napoli, Italy \\ ${ }^{g}$ Dipartimento di Fisica, Università degli Studi di Napoli "Federico II", Complesso \\ Universitario di Monte Sant'Angelo, via Cintia, I-80126, Napoli, Italy \\ ${ }^{h}$ Saitama University, Shimo-Okubo 255, Sakura-ku, Saitama-shi, 338-8570, Japan \\ ${ }^{i}$ RIKEN Advanced Science Institute, 2-1 Hirosawa, 351-0198 Wako, Japan \\ ${ }^{j}$ Universidad Nacional Autónoma de México (UNAM), Av. Universidad 3000, Copilco, \\ Coyoácan, 04510 Mexico City, Federal District, Mexico \\ ${ }^{k}$ SPace and AStroparticle (SPAS) Group, Universidad de Alcalá, Ctra. \\ Madrid-Barcelona, km. 33.7, E-28871, Alcalá de Henares, Madrid, Spain \\ ${ }^{\prime}$ ISDC Data Center for Astrophysics, University of Geneva, Chemin d'Ecogia 16, \\ CH-1290, Versoix, Switzerland \\ ${ }^{m}$ http://jemeuso.riken.jp
}

\section{Abstract}

In this paper we describe the expected performance of JEM-EUSO. Designed

*Corresponding authors

Email address: bertaina@to.infn.it (M. Bertaina)

${ }^{1}$ Now at: Potsdam Institute for Climate Impact Research, Research Domain I - Earth System Analysis, Telegrafenberg A62, D-14473 Potsdam, Germany 
as the first mission to explore the ultra-high energy universe from space, JEM-EUSO will monitor the Earth's atmosphere at night to record the UV (300-430 nm) tracks generated by the extensive air showers produced by ultra-high energy primary particles developing in the atmosphere. Studies of the expected aperture, the estimated exposure, as well as on the methods to infer arrival direction, energy, and $X_{\max }$ of primary particles are presented.

Keywords: UHE Cosmic Rays, Space Instrumentation, JEM-EUSO, Extensive Air Showers

PACS: : 98.70.Sa, 95.85.Ry, 96.50.sd

\section{Introduction}

The Extreme Universe Space Observatory (EUSO) onboard the Japanese Experiment Module (JEM) of the International Space Station (ISS), JEMEUSO, (Ebisuzaki et al., 2008; Takahashi et al., 2009; Casolino et al., 2011; Ebisuzaki et al., 2013) is an innovative space-based mission with the aim of detecting Ultra-High Energy Cosmic Rays (UHECR) from the ISS, by using the Earth's atmosphere as a calorimeter viewed by a fluorescence detector. An observatory able to produce an arrival direction map with more than several hundreds events above $\sim 5 \times 10^{19} \mathrm{eV}$ would give important information on the origin of the UHECRs and identify structures in the sky map that contain information about the source density and/or distribution. This is likely to lead to an understanding of the acceleration mechanisms potentially producing discoveries in astrophysics and/or fundamental physics. The comparison of the energy spectra among the spatially resolved individual sources will help to clarify the acceleration and emission mechanisms, and also significantly strengthen the confirmation of the Greisen-ZatsepinKuz'min (Greisen , 1966; Zatsepin and Kuz'min, 1966) process for the validation of Lorentz invariance up to Lorenz factor of $\sim 10^{11}$. Neutral components, i.e. neutrinos and gamma rays, can be detected as well, if their fluxes are high enough.

JEM-EUSO consists of a UV telescope and of an atmospheric monitoring system. Orbiting the Earth every 90 minutes at an altitude of around $400 \mathrm{~km}$ from Earth's surface ${ }^{2}$, JEM-EUSO is designed to detect UV (300-430

\footnotetext{
${ }^{2}$ Hereafter, Earth's surface is referred to as the assumed Earth's ellipsoid model and the altitude is measured from this level.
} 
nm) fluorescence photons produced along the track of Extensive Air Showers (EAS) in the atmosphere. The telescope optics consists of three double-sided curved circular Fresnel lenses with $2.65 \mathrm{~m}$ maximum diameter. The minimum diameter of the lenses is $1.9 \mathrm{~m}$ due to size constraints in one dimension. This shape is referred to as 'side-cut' and is required to satisfy constraints of the H-IIB Transfer Vehicle (HTV) which will transport the JEM-EUSO telescope to the ISS (Zuccaro Marchi et al., 2011). The dimension of the Field-of-View $(\mathrm{FoV})$ are around $64^{\circ}$ and $45^{\circ}$ on the major and minor axes, respectively. For these axes, the projected lengths on Earth's surface are around $500 \mathrm{~km}$ and $330 \mathrm{~km}$, respectively, for an orbit altitude of $400 \mathrm{~km}$. The effective solid angle is $\sim 0.85 \mathrm{sr}$. The telescope records the EAS-induced tracks with a time resolution of $2.5 \mu$ s called Gate Time Unit (GTU) and an angular resolution of $0.074^{\circ}$ (corresponding to a spatial resolution of around $0.5 \mathrm{~km}$ on the Earth's surface at the nadir) by using a highly pixelized Focal Surface (FS) detector with around $3 \times 10^{5}$ pixels in total (Kajino et al., 2011). These timesegmented images allow the measurement of the energy and arrival direction of the primary particles.

Since the ISS orbits the Earth in the latitude range $\pm 51.6^{\circ}$, moving with a ground speed of the sub-satellite point of $\sim 7 \mathrm{~km} \mathrm{~s}^{-1}$, the variability of the FoV observed by JEM-EUSO is much higher than that observed by ground-based experiments. In particular, the atmospheric conditions, which eventually determine the aperture, must be carefully monitored via the atmospheric monitoring system consisting of an infrared camera (Rodríguez Frías M.D. et al., 2013) and a LIDAR (Neronov et al., 2011).

Thanks to the ISS orbit, JEM-EUSO monitors with a rather uniform exposure both hemispheres, minimizing the systematic uncertainties that may affect any comparison between independent observatories exploring, from ground, different parts of the sky.

Another peculiarity of JEM-EUSO, in comparison to any existing or planned ground-based observatory, is the significant increase in the aperture (see Section 4). There are, however, other interesting aspects in using space-based UHE observatories. First, the almost constant distance of the EAS to the detector considerably reduces problems associated with the determination of the solid angle and with the different attenuation suffered by the UV light in the atmosphere (Takahashi et al., 2009). Second, the observation from space significantly reduces uncertainties due to scattering by aerosols limited to altitudes in the planetary boundary layer. Finally, as the EAS maximum occurs, for most zenith angles, at altitudes higher than $\sim 3 \mathrm{~km}$ from Earth's 
surface, space measurements are also possible in some cloudy conditions, i.e. when the cloud-top is located below the shower maximum. Compared to ground-based detectors, the cloud impact can be less severe, e.g. space-based observations can view extended air showers over low altitude clouds.

The JEM-EUSO observational approach mainly relies on the fact that a substantial fraction of the UV fluorescence light generated by the EAS can reach a light-collecting device of several square meters located at several hundreds kilometers away from the EAS track (Pallavicini et al., 2012). Typically a few thousand photons reach the JEM-EUSO detector for a shower produced by a $10^{20} \mathrm{eV}$ particle. JEM-EUSO is designed to record not only the number of photons but also their direction and time of arrival. It is the observation of the specific space-time correlation of the signal that allows to identify EAS tracks very precisely in the night glow background. The atmospheric depth of the shower maximum $X_{\max }$ is directly linked to the nature of the primary, while the arrival direction and energy of the EAS can be used to determine the arrival direction maps.

We note that JEM-EUSO has considerably improved with respect to the original Extreme Universe Space Observatory (EUSO Collaboration, 2003) studied by the European Space Agency. Main improvements have to be ascribed to the new optics, to the photo-detector $(\sim 1.6$ times higher detection efficiency of the Ultra Bi-alkali Photomultiplier Tube), to the better geometrical layout of the photo-detector on the FS that maximizes the active area of the detector by reducing the dead zones (gaps) on the FS, and to the improved performance of the electronics, which allows to exploit more complex trigger algorithms.

The outline of the paper is as follows: Section 2 is devoted to the description of the ESAF simulation code used to evaluate the performance of the instrument and to develop the reconstruction techniques presented in the paper. In Section 3 we discuss the estimation of the observational duty cycle, local light effects and the role of clouds. Computation of the aperture for both clear and cloudy conditions is described in Section 4, together with the exposure in the nadir observation (nadir mode). The paper follows in Section 5 with the description of the pattern recognition techniques used to extract the signal information from the background when analyzing air shower tracks, and in Section 6 with a detailed description of the algorithms used to reconstruct the main parameters of the air shower track: arrival direction, energy and $X_{\max }$. Examples of results obtained for subclasses of events in clear-atmosphere conditions are presented in Section 7. 


\section{JEM-EUSO Simulation and Analysis Framework}

The EUSO Simulation and Analysis Framework (ESAF; (Berat et al., 2010)) is currently used as the simulation and analysis software for the JEMEUSO mission. It had been developed in the framework of the ESA-EUSO mission. ESAF performs the simulation of the shower development, photon production and transport in atmosphere, and detector simulations for optics and electronics. Furthermore, algorithms and tools for the reconstruction of the shower properties are included in the ESAF package. During the JEMEUSO Phase-A study, all the necessary steps were taken to implement the JEM-EUSO mission configuration in order to assess the mission performance. ESAF is subdivided in two main parts: Simulation and Reconstruction.

\subsection{The Simulation framework}

The Simulation Framework is meant to simulate all the physical processes which are related to the shower development, the light production and propagation, the detector and eventually the telemetry.

Several shower simulators are implemented in ESAF, following parametric and Monte Carlo approaches. As parametric generator, the GreisenIlina-Linsley (GIL) function (Ilina et al., 1992), is used to reproduce the profile for hadronic EASs generated by CORSIKA (Heck et al., 1998) with QGSJet-01 (Ostapchenko et al., 2006) interaction model. Other generators such as the Conex simulator (Bergmann et al., 2007) are interfaced with ESAF. The interface for external generators allows to simulate also neutrinos (Guzmán et al., 2013) and gamma rays. LIDAR events can be simulated as well (Neronov et al., 2013), while the effects of other sources of light such as lightnings, cities and meteors can be reproduced at a first order of approximation by means of test light sources.

Both fluorescence and Cherenkov light productions are taken into account in ESAF. The fluorescence spectrum is simulated according to (Nagano et al., 2004). Both the reflected and the back-scattered Cherenkov light components are considered. The Cherenkov reflection is treated according to a Lambertian reflector. Therefore, all the photons are reflected diffusely due to the very irregular terrestrial surface. In the present simulation an albedo of $5 \%$ is considered for all events. This is a typical value assumed in literature for water and forests. However, in case of land the value typically varies from $2 \%$ on savannah to $85 \%$ on fresh snow. All the photons are affected 
by Rayleigh scattering and ozone absorption. Optionally, clouds can be simulated as a constant layer of variable altitude thickness and optical depth. Non-uniform cloud coverage is also included in ESAF.

Once the photons reach the detector they are taken over by the optics module. Several optics simulation approaches have been considered. The simplest and fastest one is a parametric simulation module that calculates analytically the position of the photon on the FS and adds a Gaussian spread around this position. This is intended to be a fast working tool to test the features of the different optics designs in an approximated way. Second, a full ray-trace code used in the actual optics design and development (Zuccaro Marchi et al., 2011) is implemented in ESAF that allows every photon to be simulated within the optics. In this work, we employ this option for simulation. Third, a GEANT4 (Agostinelli et al., 2003) optics module is available as well (Biktemerova et al., 2009). Composition of the photon spectrum arriving at the telescope entrance, i.e. pupil, is analyzed, and is shown in Figure 1. Direct fluorescence light as well as reflected and back-scattered Cherenkov light is visible. The contribution from the back-scattered Cherenkov (plotted on top of the fluorescence one) corresponds only to a limited fraction of the total signal. This is due to the different spectra of each signal and wavelength dependence of the optics and focal plane detector, together with the fact that no direct Cherenkov light is detectable.

Moreover, the same figure shows the event through the entire detector from the pupil to detected counts regardless of the photon's origins.

Once the photons reach the FS they are transported through the filter and the optical adapter before reaching the photocathode. All the relevant effects including geometrical losses, inefficiencies of the adapter and of filter (Schott BG3, (Schott, 2008)) are taken into account. A parametrization of the MultiAnode Photomultiplier Tube (MAPMT) is included in the electronics part. All the effects like quantum efficiency, dependence on the incident angle of photon, collection efficiency and cross talk are also taken into account pixel by pixel within one MAPMT (Kawasaki et al., 2006; Kawasaki, 2011). The implemented MAPMT is R11265-03-M64 by Hamamatsu Photonics having $8 \times 8(=64)$ pixels each of which has a dimension about $2.9 \mathrm{~mm} \times 2.9 \mathrm{~mm}$. The signal is then amplified by a parametrized gain and the resulting output current is collected and treated by the Front End Electronics (FEE) module. A threshold is set on the MAPMT output current in order to accept or reject the signal count. The main parameters of the JEM-EUSO telescope are summarized in Table 1. The right plot of Figure 1 shows the result of the 

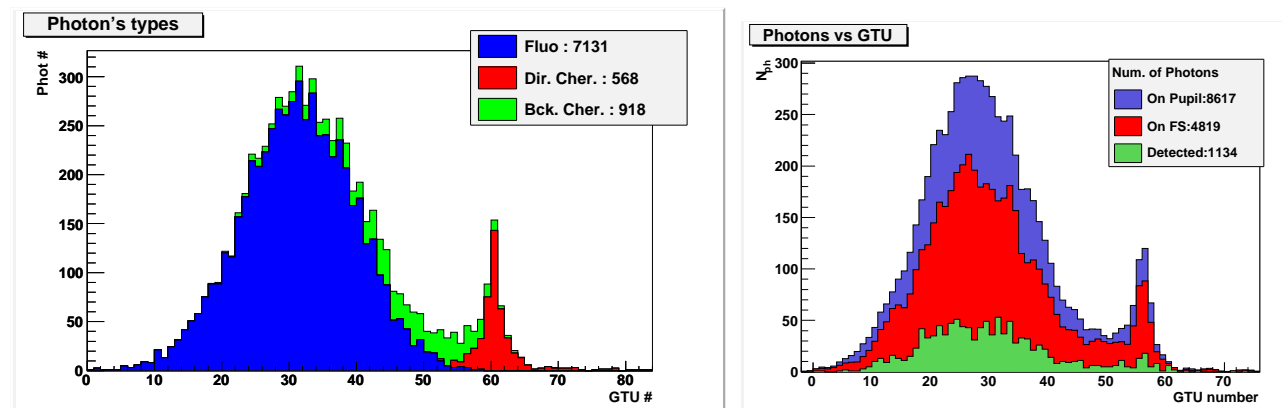

Figure 1: Left: Composition of the photons as a function of time at the detector pupil for a $10^{20}-\mathrm{eV}$ shower $60^{\circ}$ zenith angle, as simulated by ESAF with the GIL parametrization. Blue (green) color indicates the contribution from fluorescence (back-scattered Cherenkov) light; red color in the legend is meant for the reflected Cherenkov light from ground. The contribution from the back-scattered Cherenkov is plotted on top of the fluorescence one. It corresponds only to a limited fraction of the total signal. This is due to the different spectra of each signal and wavelength dependence of the optics and focal plane detector, together with the fact that no direct Cherenkov light is detected. Right: Same event as seen by the detector. The blue (red) curve gives the number of photons at the pupil (focal surface). The green one represents the counts finally detected at front-end electronics level. The numbers in the legend indicate the integral counts in each of the histograms. The plots are obtained using a ray-trace simulation of the optics and they apply to clear atmosphere.

simulated response of the different parts of the detector as implemented in ESAF for the simulated air shower shown in the left part of the same figure.

One of the roles of the trigger algorithm is to filter the background in order to increase the signal-to-noise ratio. Being telemetry limited, the instrument cannot afford the transmission of the entire FS data to the Earth. The entire triggering scheme is therefore organized in a multiple step filtering. After the FEE identifies a photon count a first search for persistency is done at the level of a Photo-Detector Module ${ }^{3}$ (PDM). This is called the first level trigger. After a trigger signal is issued at this level, data are sent at the next level named Cluster Control Board ${ }^{4}$ trigger. This is also called second level trigger or Linear Track Trigger (LTT). Here the background trigger rate must be further reduced to fit within the telemetry constraints $(0.1 \mathrm{~Hz}$ on the entire FS). Once this level of the trigger is issued, the data of triggered events are sent to the reconstruction framework. A more comprehensive review on

\footnotetext{
${ }^{3}$ Part of the FS consisting of 36 MAPMTs. The FS is filled with 137 PDMs.

4 Electronics board which operates on $\sim 300$ MAPMTs.
} 
Table 1: Parameters of the JEM-EUSO telescope. The values in parentheses apply at the edge of the FoV, otherwise at the the center of the FoV. The ensquared collection efficiency is the ratio of the number of photons focused within a pixel area to those incident on the entrance aperture of the optics. The ensquared energy is the ratio of photons focused within the area of a pixel to those reaching the photo-detector on the FS.

\begin{tabular}{l|r|l}
\hline Parameter & Value & Note \\
\hline Optics & $4.5 \mathrm{~m}^{2}$ & baseline \\
Optical aperture & $35 \%(15 \%)$ & for $\lambda=350 \mathrm{~nm}$ \\
Ensquared collection efficiency & $86 \%(80 \%)$ & for $\lambda=350 \mathrm{~nm}$ \\
Ensquared energy & $300-430 \mathrm{~nm}$ & \\
Optical bandwidth & $0.85 \mathrm{sr}$ & \\
Field of view & $1.4 \times 10^{5} \mathrm{~km}^{2}$ & for $H_{\text {ISS }}=400 \mathrm{~km}$ \\
Observational area (nadir mode) & $3.2 \times 10^{5}$ & \\
\hline FS detector and electronics & $0.074^{\circ}$ & \\
Number of pixels & $41 \%$ & $\lambda=350 \mathrm{~nm}$ \\
Spatial angular resolution & $80 \%$ & \\
Pixel size at ground & $<2 \%$ & \\
Quantum efficiency & $97 \%$ & for $\lambda=350 \mathrm{~nm}$ \\
Collection efficiency & $2.5 \mu \mathrm{s}$ & \\
Cross talk & $(0.61 \mathrm{~km})$ & for $H_{\text {ISS }}=400 \mathrm{~km}$ \\
Transmittance of UV filter & \multicolumn{2}{|l|}{$\begin{array}{l} \\
\text { Sampling time (GTU) }\end{array}$} \\
\hline
\end{tabular}

the trigger scheme is given in Catalano et al. (2009). The aperture curves reported in this paper take into account the application of both trigger levels and the rate of background events inside the telemetry constraints for the different background levels. It is important to emphasize here that a spacebased detector has to be much more selective than a ground-based experiment because of telemetry constraints. This is one of the key factors determining the threshold in energy. On the other side, the fraction of scientific data in the sample will be of high quality. Thus, it is expected that further quality cuts applied in the offline analysis will not cause a significant reduction of data. 


\subsection{The Reconstruction framework}

Aim of this framework is to analyze the detector response in order to identify the arrival direction, the energy and the nature of the primary particle. The first step consists in the identification of the signal inside the transmitted data. Then through fit procedures the arrival direction of the primary is calculated. Several different fit algorithms have been included in ESAF. As the last step, the shower profile, $X_{\max }$, and the energy are reconstructed. A more comprehensive review of the reconstruction modules is given in Sections 5 and 6.

\section{Estimation of observational duty cycle, local light effects and role of clouds}

The estimation of the exposure of a space-based experiment such as JEMEUSO requires accounting for: a) the characteristics of the EAS development in the atmosphere as observed from space, b) the properties of the telescope, including its orbit and FoV, c) the various sources of relatively steady background like night-glow and moonlight, d) the overall optical transmission properties of the atmosphere, in particular the possible presence of clouds, and e) the effect of anthropogenic light, atmospheric flashes such as lightning, Transient Luminous Events (TLEs) and meteors, and South Atlantic Anomaly (SAA). Items a) and b) are the principal factors determining the threshold in energy and maximum aperture of the telescope. Item c) limits the observational duty cycle of the mission. Items d) and e) affect the instantaneous aperture of the telescope. All the evaluations are performed independently. As shown in Section 4, Equation (2) explicitly describes the individual terms that quantify the duty cycle calculation. The definition of each term is reported along the sections. More extended discussions of most of the above effects are reported in (Adams et al., 2013). In the following we summarize those results and we add further analyses conducted in order to strengthen such conclusions.

\subsection{Night-glow and moonlight}

One essential parameter to estimate the JEM-EUSO exposure is the fraction of time during which EAS observation is not hampered by the brightness of the atmosphere. We denote the main component of the intensity of diffuse background light, $I_{\mathrm{BG}}$, a quantity which is variable over time. We define 
the observational duty cycle, $\eta$, the fraction of time during which the background intensity is lower than a given value $I_{\mathrm{BG}}^{\mathrm{thr}}$. Moonlight is the largest background component in the night-time observation. In case of JEM-EUSO only back-scattered moonlight is relevant, while ground-based telescopes are subject also to direct moonlight.

We estimate moonlight contamination from the phase of the Moon together with its apparent position as seen from the ISS. In our approach the ISS trajectory provided by NASA SSCweb (NASA-SSCWEB) is traced with 1-min time step and the moonlight at the top of the atmosphere is estimated according to a modified version of the technique presented in (Montanet, 2004). For every position of the ISS in the period from 2005 till 2007 the zenith angle of the Sun, and that of the Moon, as well as the Moon phase angle, are calculated. The night-time is defined as the absence of Sun in the visible sky at the orbit level. This requires the zenith angle of the Sun to be greater than $109^{\circ}$ for an orbiting altitude of $H_{\text {ISS }}=400 \mathrm{~km}$ and results in $34 \%$.

Figure 2 shows the observational duty cycle as a function of the accepted background level.

The fraction of time during which $I_{\mathrm{BG}}$ is less than 1500 photons $\mathrm{m}^{-2} \mathrm{sr}^{-1}$ $\mathrm{ns}^{-1}$ is $20 \%-21 \%$ resulting in an average background level of 550 photons $\mathrm{m}^{-2} \mathrm{sr}^{-1} \mathrm{~ns}^{-1}$. Of course the operation of the instrument is not limited by the value 1500 photons $\mathrm{m}^{-2} \mathrm{sr}^{-1} \mathrm{~ns}^{-1}$, therefore, this is a conservative estimate for the highest energies where measurement can be performed even in higher background conditions. In fact, for EAS of energy $10^{21} \mathrm{eV}$, the signal-to-noise ratio is still higher than 10 around shower maximum even for a night-glow background of 6000 photons $\mathrm{m}^{-2} \mathrm{sr}^{-1} \mathrm{~ns}^{-1}$.

In the following discussion we assume $\eta_{0} \sim 20 \%$ as the reference value for the observational duty cycle.

\subsection{Lightning and TLEs}

In addition to the diffuse sources of background, there are transient local sources such as lightning and TLEs, aurorae or city light. In the cases of lightning and TLEs, estimates of the reduction in observational duty cycle and instantaneous aperture are performed assuming the rate of events detected by Tatiana satellite (Garipov et al., 2005). We further assume that the EAS measurement is not possible in the whole FoV as long as the location of the event exists within the JEM-EUSO FoV. For a conservative estimation, we apply $\sim 70 \mathrm{~s}$ to all events, that corresponds to the maximum time that a light 


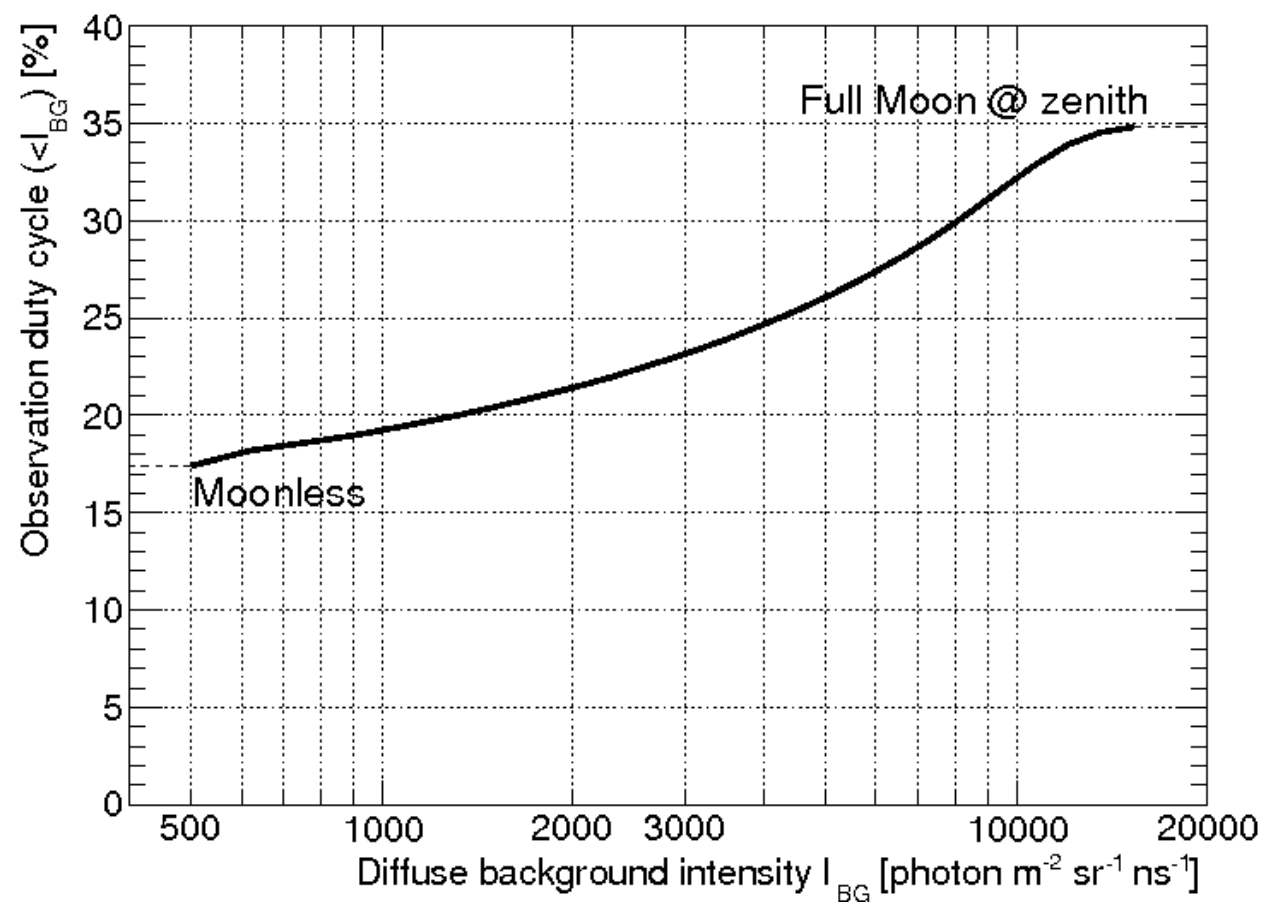

Figure 2: Observational duty cycle $\eta$ as a function of threshold background level $I_{\mathrm{BG}}^{\mathrm{thr}}$.

source takes to traverse the major axis of the FoV. In the above estimation we do not take into account the fact that lightning is very often associated with high-altitude clouds. Therefore, some double counting is included when both lightning and cloud inefficiencies are factorized in the estimation of the exposure. This is explicitly done to reinforce the conservation nature of the calculation.

\subsection{Auroral light}

Auroras taking place in the FoV of the detector very likely restrain EAS measurements, therefore, they have to be considered as a factor that reduces the observational duty cycle. The method to evaluate the fraction of time in which JEM-EUSO will face auroral light is described in Appendix A.

The fraction of time in which EAS measurements cannot be performed due to the presence of auroral light $\left(f_{\mathrm{AL}}\right)$ from 2000 to 2011 is presented in 

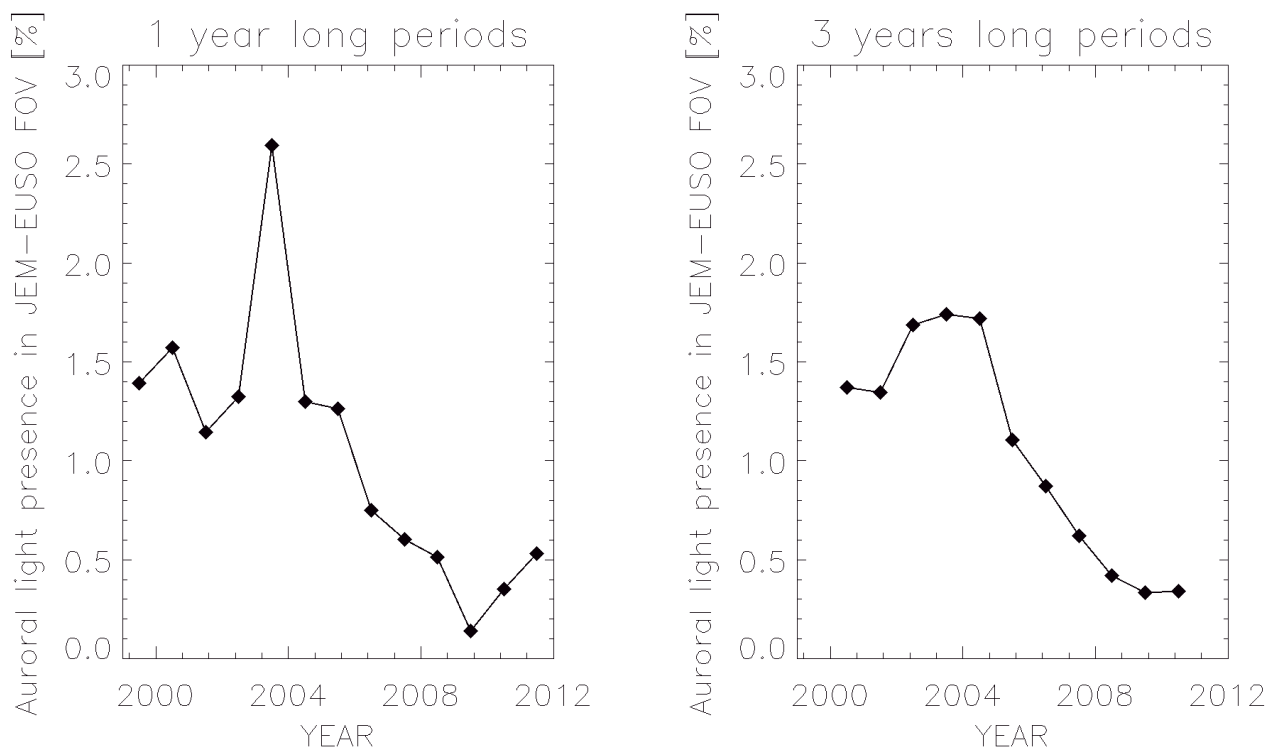

Figure 3: Left panel: Fraction of time $f_{\mathrm{AL}}$ in which auroral light restrain EAS measurements for one year long periods from 2000 to 2011. Right panel: Same as in the left panel, but integrating for 3-year periods.

the left panel of Figure 3. $f_{\mathrm{AL}}$ varies between $2.59 \%$ in year 2003 to $0.14 \%$ in year 2009. In this estimation it is assumed that the observation of EASs can not be performed in all the PDMs which have at least one pixel illuminated by auroral light.

Since the effect clearly depends on time, specifically on solar cycle, the influence is estimated also in 3 years moving time windows, i.e. periods of 2000-2002, 2001-2003, and 2009-2011. We select 3-year long periods because we estimate similarly long measurements of JEM-EUSO on ISS in years 2017 2019. The effect of aurorae presented in the right panel of Figure 3 is the highest for periods 2002-2004 and 2003-2005 when $f_{\mathrm{AL}} \sim 1.7 \%$ and it is lowest in periods 2008-2010 and 2009-2011 when $f_{\mathrm{AL}} \sim 0.4 \%$.

To evaluate the influence in years 2017-2019 we should estimate geomagnetic conditions during such period. The conservative estimation is, that JEM-EUSO will face declining solar activity and solar minimum somewhere during measurement period. In that case we estimate an effect of auroral light to restrain the EAS measurement for $\sim 1 \%$ of the time which has to be 
added to the other factors quantified in this section.

\subsection{Southern Atlantic Anomaly}

Three different sources of specific UV background are considered in the South Atlantic Anomaly (SAA): a) the UV background created by galactic cosmic rays interacting in the atmosphere; b) airglow production in SAA; c) interaction of electrons trapped in the Earth radiation belt with the JEMEUSO detector, namely the optics, where relativistic electrons can create Cherenkov photons in the lenses. A detailed description of the methods and results is reported in (Mernik et al., 2013). Here the main conclusions are summarized.

Regarding the UV background created by the galactic cosmic rays, it is found that such contribution is negligible, being a few orders of magnitude lower than the night-glow background.

By means of the AURIC model (Strickland et al., 1999), which is a computational tool for upper atmospheric radiation, capable of computing day-glow and night-glow radiation for many spectral features, radiations in the 300$400 \mathrm{~nm}$ wavelength range for Herzberg- I and II and Chamberlain radiation is calculated. The purpose of this study is to estimate the relative intensity of the UV night-glow radiation in SAA compared to the rest of the Earth. The analysis shows that the UV background emission in SAA is in the range of values observed in the rest of the Earth.

Regarding the UV emission in the detector lenses due to trapped electrons, the SPENVIS AE-8 model (Heinderickx et al., 2001) is used to estimate the intensity of the trapped electrons along the ISS trajectory. Figure 4 shows the intensity distribution of electrons with energy $E>40 \mathrm{keV}$. The SAA, as well as the region over North America, shows higher intensities.

The maximum intensities in the center of SAA are of the orders of $\sim$

$10^{6}$ electrons $\mathrm{cm}^{-2} \mathrm{~s}^{-1}$. The spectrum of trapped electrons in the center of the SAA is used as an input for simulation of relativistic electrons crossing the detector lenses (see Figure 5). The detector response is obtained by a full GEANT4 simulation incorporating a complete description of the Fresnel lenses based on (Biktemerova et al., 2011). Results show a conversion factor between electrons and photons of the order of $\sim 0.1$. This is due to the fact that only electrons with energy $E \gtrsim 600 \mathrm{keV}$ contribute to the generation of Cherenkov photons. This results in an expected flux of $\sim 4$ photon $\mathrm{m}^{-2} \mathrm{~ns}^{-1}$ to be compared with about $400-500$ photons $\mathrm{m}^{-2} \mathrm{~ns}^{-1}$ due to the standard night-glow background within the $0.85 \mathrm{sr}$ of the optics. This indicates that 


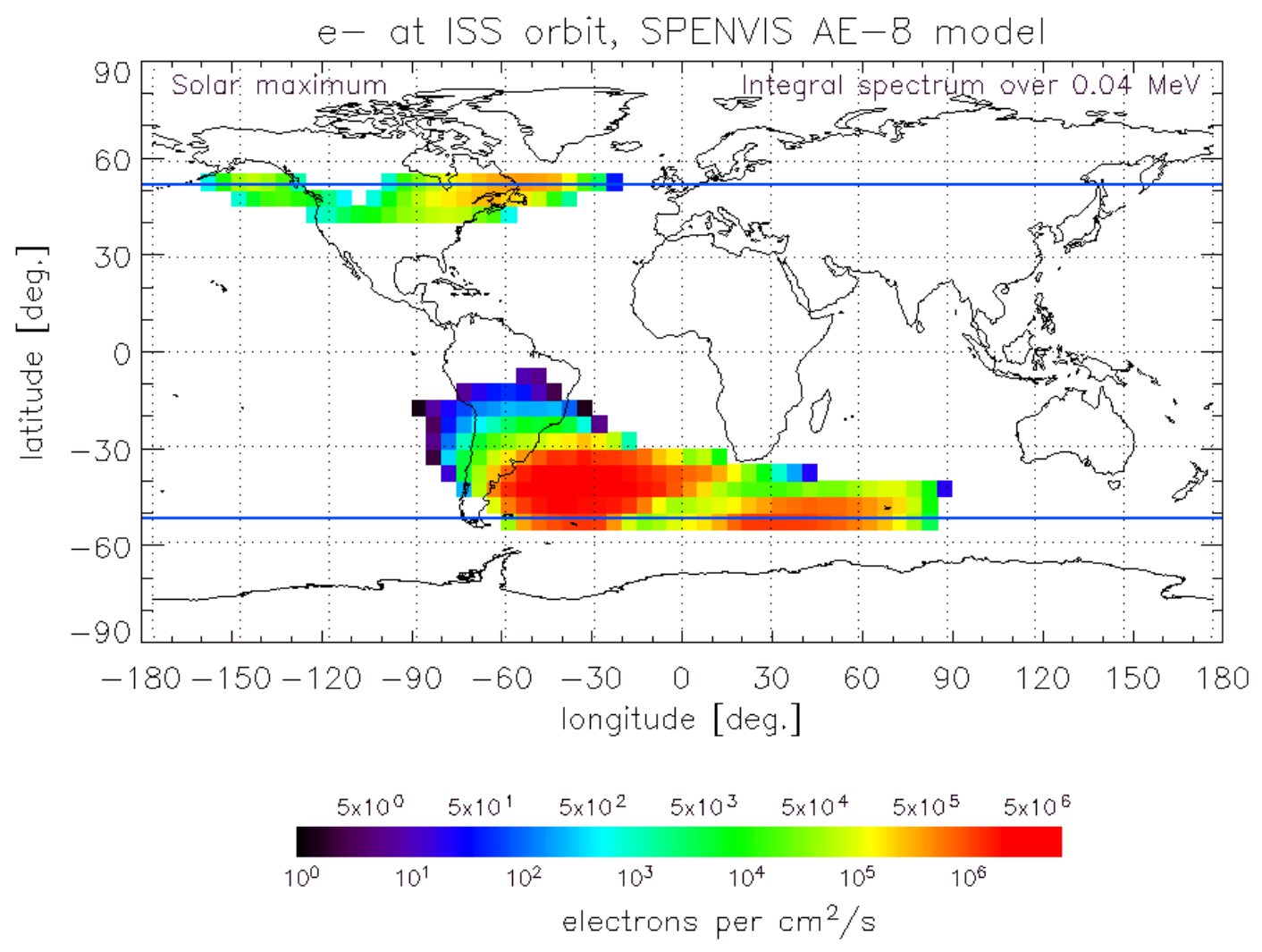

Figure 4: Intensities of the trapped electrons evaluated from SPENVIS AE-8 model for solar maximum period. Blue lines show the ISS trajectory borders at geographical latitudes $\pm 51.6^{\circ}$ within which the evaluation is performed.

the flux of photons due to trapped electrons in non disturbed magnetosphere is negligible compared to the average UV background level measured by the detector. In case of disturbed magnetosphere the electron flux would increase by $\sim 2$ orders of magnitude. Therefore, it will double the average background level. However, it has to be reminded that JEM-EUSO is expected to fly in the years 2017-2019, therefore, the amount of disturbances should be not particularly high for the same considerations done in the case of aurorae.

\subsection{Anthropogenic light}

To evaluate the effect of the stationary light sources on the Earth, which are mainly anthropogenic, we use the Defense Meteorological Satellite Program (DMSP) (NASA-NOAA) database. Annual averages of light intensities 


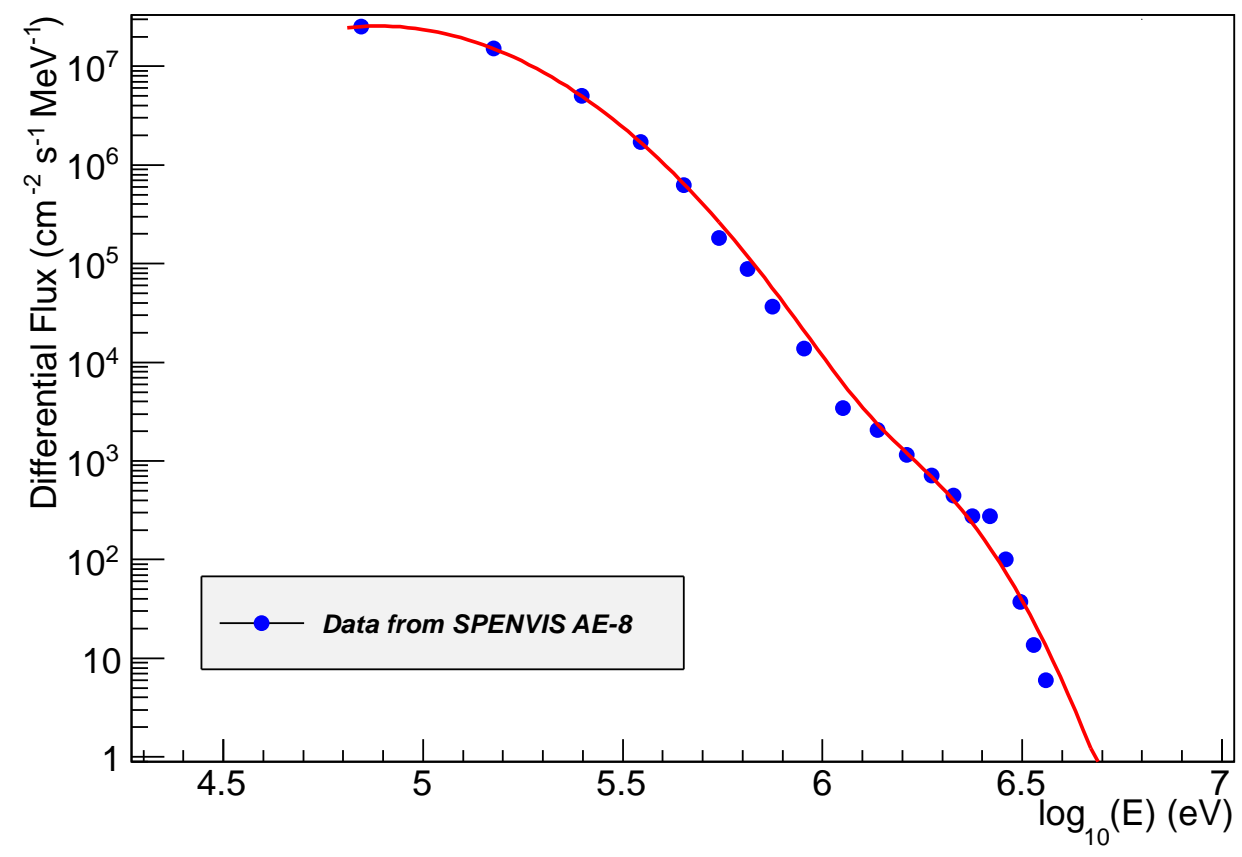

Figure 5: Differential flux of trapped electrons evaluated from SPENVIS AE-8 model for solar maximum period in the central region of the SAA. The continuous line represents the parametrization used for the simulation.

for cloud-free moonless nights are used to estimate the presence of local light along the ISS trajectory. The DMSP data provide the light intensity in 64 different levels on a 30-arcsecond grid on latitude and longitude in the wavelength range $350 \mathrm{~nm}-2 \mu \mathrm{m}$. The units are arbitrary, with equally spaced steps. The stationary background is dominated by visible light. In the following, we make the conservative assumption that no measurement of EASs is performed if, in a region viewed by a PDM, there is at least one pixel which detects a light intensity which exceeds the average level by a factor of 3 or more (higher than 7 in DMSP units). The average level of intensity (2.6 in DMSP units) corresponds essentially to the typical condition on oceans. With this assumption the inefficiency of the instantaneous aperture is of $\sim 7 \%$. It is important to remember that Tatiana measurements (Garipov et al., 2005), without focusing optics, indicate 2-3 times higher intensities in UV 
Table 2: Comparison of the TOVS, ISCCP and CACOLO databases for the relative cloud occurrence in different meteorological situations. The data correspond to daytime, with a weighted average between land and ocean. The classification of the TOVS and ISCCP data is based on the cloud-top $\left(H_{\mathrm{C}}\right)$ altitude while for CACOLO it is based on the cloud-bottom. The abbreviation are defined as follows, HC: High Clouds $\left(H_{\mathrm{C}}>6.5 \mathrm{~km}\right)$; MC: Middle Clouds $\left(H_{\mathrm{C}}=3.2-6.5 \mathrm{~km}\right)$, LC: Low Clouds $\left(H_{\mathrm{C}}<3.2 \mathrm{~km}\right)$, CA: Clear Atmosphere.

\begin{tabular}{l|cc||c}
\hline atmospheric & \multicolumn{3}{|c}{ Database } \\
\cline { 2 - 4 } condition & TOVS & ISCCP & CACOLO \\
\hline HC & $30 \%$ & $23 \%$ & $18 \%$ \\
MC & $12 \%$ & $16 \%$ & $25 \%$ \\
LC & $30 \%$ & $26 \%$ & $40 \%$ \\
CA & $28 \%$ & $35 \%$ & $17 \%$ \\
\hline
\end{tabular}

above big cities such as Mexico City and Houston compared to the average background level over the ocean. Finally, by combining the above estimations for lightnings $(\sim 2 \%)$, aurorae $(\sim 1 \%)$ and DMSP data the overall loss of coverage is $f_{\text {loc }} \sim 10 \%$.

\subsection{Role of clouds}

In order to quantify the reduction on the effective instantaneous aperture of the telescope due to the presence of clouds, a study on the distribution of clouds as a function of altitude, optical depth and geographical location are performed using TOVS, ISCCP and CACOLO meteorological data sets (TOVS; ISCCP; CACOLO) obtaining similar results on the cloud occurrence. Table 2 shows a comparison of cloud distribution in the troposphere among the three datasets where land and ocean data are combined in a weighted average. Only daytime data are shown since CACOLO employs visual observation.

Significant differences exist among the three databases for each of the four categories shown in the table. However, if one considers the good cases for JEM-EUSO, namely low altitude clouds or clear atmosphere, the three datasets are in reasonable agreement, with a minimum of $57 \%$ for CACOLO to a maximum of $61 \%$ in case of ISCCP. Differences among the datasets may be due to the different locations of the instruments (at ground in case of CACOLO) and to the different bandwidth used in the analysis. Since the TOVS data show the largest fraction of high clouds, which are the most 
critical in case of EAS observation from space, estimates of the fraction of EASs measurable by JEM-EUSO using the TOVS data can be considered conservative.

A comparison between day and night cloud coverage is performed for clouds above land as higher variations are expected in comparison with the day-night variation above the ocean. Slight differences exist, typically $\sim 5 \%$, though the general trend seems to be independent of the geographical and temporal conditions. High clouds are particularly frequent in the equatorial and tropical regions. This is expected, due to the presence of the cloudiness associated to the Inter Tropical Convergence Zone. More details on these results are reported in (Adams et al., 2013) and references therein.

Then, the matrix of cloud occurrence are determined as a function of the optical depth and cloud-top altitude using the TOVS data. Showers are simulated using ESAF according to the matrix of cloud occurrence determining the trigger efficiency in the different conditions, and obtaining the corresponding aperture. Results (Sáez-Cano et al., 2013) show that the ratio between the aperture when the role of clouds is included compared to purely clear atmosphere, for those events which have 'good quality' characteristics (clouds with optical depth $<1$, or shower maximum above the cloud-top altitude) is $\sim 72 \%$ almost independently of energy. This result is comparable to a previous estimation done at the time of the EUSO experiment and is a bit higher because stricter quality cuts were applied in (Berat et al., 2010).

The exposure distribution has high degree of uniformity in declination and right ascension. Local and seasonal dependence of the cloud distribution, as well as anthropogenic light and the different twilight duration for different latitudes introduce only a $\pm 10 \%$ non uniformity in the exposure distribution over the celestial sphere (Shinozaki et al., 2013).

\section{Aperture and exposure curves in nadir mode}

The last parameter needed to estimate the aperture and the exposure is the trigger efficiency. Main objective of the trigger system is to reduce the rate of triggers due to background fluctuations to $\sim 0.1 \mathrm{~Hz}$ on FS that is imposed by downlink telemetry capabilities. The rejection level of the trigger algorithm determines the aperture of the instrument as a function of the energy. The rejection power depends also on the average night-glow background. 


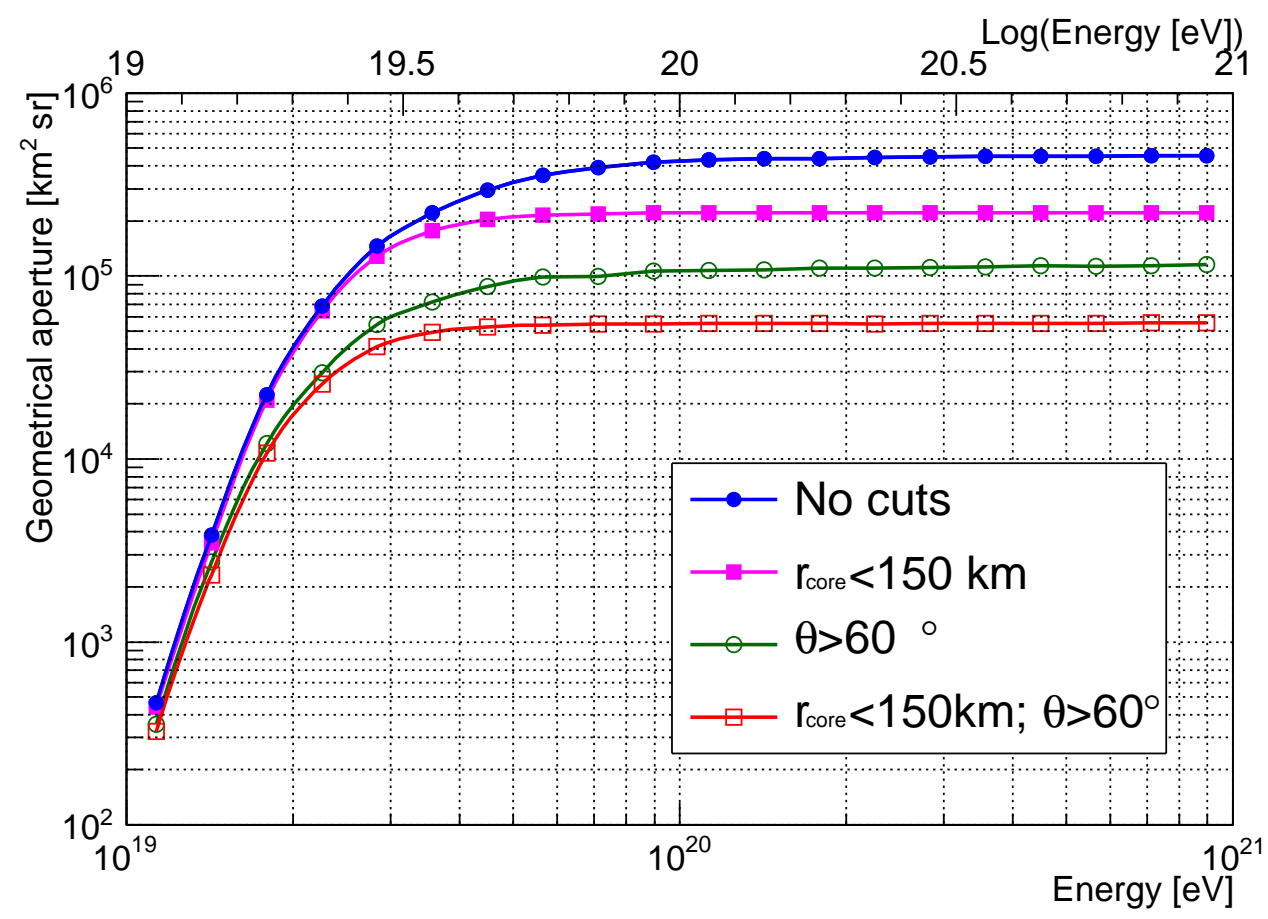

Figure 6: Geometrical aperture as a function of energy. The filled circles and squares indicate geometrical apertures for the entire observation area and $r_{\text {core }}<150 \mathrm{~km}$ respectively. The open circles and squares include a zenith angle cut of $\Theta>60^{\circ}$.

To estimate the geometrical aperture, $\sim 400,000$ EASs with an $E^{-1}$ spectrum are simulated using ESAF by uniformly injecting them over an extended area $S_{\text {inject }}$ in a clear-atmosphere condition for nominal background level of $I_{\mathrm{BG}}=500$ photons $\mathrm{m}^{-2} \mathrm{sr}^{-1} \mathrm{~ns}^{-1}$.

For $N_{\text {trig, }}$, triggering samples among $N_{\text {inject }}$, simulated EAS events with an energy $E$, the corresponding geometrical aperture $A(E)$ is defined by the following relation:

$$
A(E)=\frac{N_{\text {trig }}}{N_{\text {inject }}} \cdot S_{\text {inject }} \cdot \Omega_{0},
$$

where $\Omega_{0}=\pi$ sr is the solid angle acceptance for $0^{\circ}<\Theta<90^{\circ}$. By applying simple cuts on the distance $r_{\text {core }}$ from the center of FoV of the impact location of the EAS, and on the lower limit $\Theta$, lower geometrical apertures are obtained. 
Figure 6 shows the geometrical aperture as a function of simulated energy for $H_{\text {ISS }}=400 \mathrm{~km}$ along with the apertures for different geometrical cuts in $\Theta$ and $r_{\text {core }}$, distance of core location on the Earth's surface from the center of FoV. In all the following plots we refer to the simulated energies, not the reconstructed ones.

The aperture without geometrical cut reaches the plateau ${ }^{5}$ above $\sim(6-$ 7) $\times 10^{19} \mathrm{eV}$. At the highest energies, the geometrical aperture is close to saturation. The value is mainly determined by the observation area for a given $H_{\text {ISS }}$ and, therefore, higher altitudes result in the larger saturating apertures. Due to a minor contribution of EAS crossing the FoV, the geometrical aperture grows slightly with energy.

By applying the cut $\Theta>60^{\circ}$, which reduces the effective solid angle acceptance to $\pi / 4 \mathrm{sr}$, a constant aperture is achieved above $\sim(4-5) \times 10^{19} \mathrm{eV}$. In addition, a more stringent cut on core location of showers with $r_{\text {core }}<$ $150 \mathrm{~km}$ extends the constant aperture range down to $\sim 3 \times 10^{19} \mathrm{eV}$. The possibility to extend the plateau region at lower energies for a subset of events will allow a cross-check of the flux measured by the full sample of events in the specific range of energies where the aperture of the instrument has not reached the plateau level yet. Consequently, the overlapping energy range between JEM-EUSO and ground-based observatories will be enlarged.

From the above results, the exposure per year of operation for events that trigger JEM-EUSO, defined as the 'annual exposure' is evaluated as a function of energy:

$$
\text { (Annual exposure }) \equiv A(E) \cdot \kappa_{\mathrm{C}} \cdot \eta_{0} \cdot\left(1-f_{\mathrm{loc}}\right) \cdot(1[\mathrm{yr}])
$$

In this estimation, we use the cloud efficiency $\kappa_{\mathrm{C}}=72 \%$, the observational duty cycle $\eta_{0}=20 \%$, and the inefficient fraction of the aperture because of local light $f_{\text {loc }}=10 \%$, respectively, which leads to an overall conversion factor from geometrical aperture to exposure of $\sim 0.13 \mathrm{yr}(=0.72 \times 0.2$ $\times 0.9$ ). The operational inefficiencies related to ISS (rockets docking on ISS, lid operation, detector maintenance or aging, etc.) as well as quality cuts on reconstruction are not taken into account yet, and will be addressed in future. Therefore, the present results constitute an upper limit on the effective exposure of the instrument for the assumed conditions.

\footnotetext{
${ }^{5}$ It is defined by the condition in which the geometrical aperture is $>0.8 \cdot S \cdot \Omega$ for the area $S$ and solid angle acceptance $\Omega$ defined by specific geometrical cuts.
} 
In the analysis described so far, a constant background level of $\left\langle I_{\mathrm{BG}}\right\rangle=$ 500 photons $\mathrm{m}^{-2} \mathrm{sr}^{-1} \mathrm{~ns}^{-1}$ is assumed. However, the background is variable with time. To take into account the effective background variation, the exposure over the time when $I_{\mathrm{BG}}<I_{\mathrm{BG}}^{\mathrm{thr}}$ given as a function of $E$ is approximated by the following relation:

$$
\text { (Overall exposure }) \propto \int_{0}^{I_{\mathrm{BG}}^{\mathrm{thr}}} A\left(\sqrt{\frac{\left\langle I_{\mathrm{BG}}\right\rangle}{I_{\mathrm{BG}}}} \cdot E\right) \cdot p\left(I_{\mathrm{BG}}\right) d I_{\mathrm{BG}},
$$

where $p\left(I_{\mathrm{BG}}\right)$ is the probability density function of $I_{\mathrm{BG}}$ according to Figure 2 . $p\left(I_{\mathrm{BG}}\right)$ indicates also the relative fraction of time spent in the interval of background intensities between $I_{\mathrm{BG}}$ and $I_{\mathrm{BG}}+d I_{\mathrm{BG}}$.

Figure 7 shows the exposure as a function of $E$ for various maximum allowed background levels obtained by convolving the trigger probability at a specific fixed background level with the fraction of time during which such background level occurs. The trigger system is capable of dynamically adjusting the thresholds to cope with variable background intensity. The trigger efficiency curve scales in energy approximately in a proportional way to $\sim \sqrt{I_{\mathrm{BG}}}$ because it depends on the Poissonian fluctuations of the average background level.

The exposure obtained with a fixed $I_{\mathrm{BG}}$ of 500 photons $\mathrm{m}^{-2} \mathrm{sr}^{-1} \mathrm{~ns}^{-1}$ is essentially equivalent to the one obtained from Equation (3) when the integration of $I_{\mathrm{BG}}$ is extended up to 1600 photons $\mathrm{m}^{-2} \mathrm{sr}^{-1} \mathrm{~ns}^{-1}$. At high energies there is still some margin of gain if a higher level of background is accepted (see i.e. the curve at less than 6300 photons $\mathrm{m}^{-2} \mathrm{sr}^{-1} \mathrm{~ns}^{-1}$ ). This is particularly useful to explore the extreme energy range where the flux rapidly decreases with energy. In any case, all the conclusions obtained in this paper are derived assuming only the standard condition of 500 photons $\mathrm{m}^{-2} \mathrm{sr}^{-1} \mathrm{~ns}^{-1}$ constant background level. In such a condition, the JEMEUSO annual exposure for the full sample of data is expected to be $\sim 9$ times larger than that of the Pierre Auger Observatory (Abraham et al., 2010) with the corresponding annual exposure of about $7000 \mathrm{~km}^{2} \mathrm{sr}$ yr, at energies around $10^{20} \mathrm{eV}$. We remind that the ratio of the full geometrical apertures between JEM-EUSO and Auger is $\sim 50$.

It is worthwhile remembering here that the aperture and exposure are derived with specific assumptions on the detector properties, background level, shower development in atmosphere, etc. All the systematic uncertainties that would increase or decrease the collected light at telescope level, either for the 


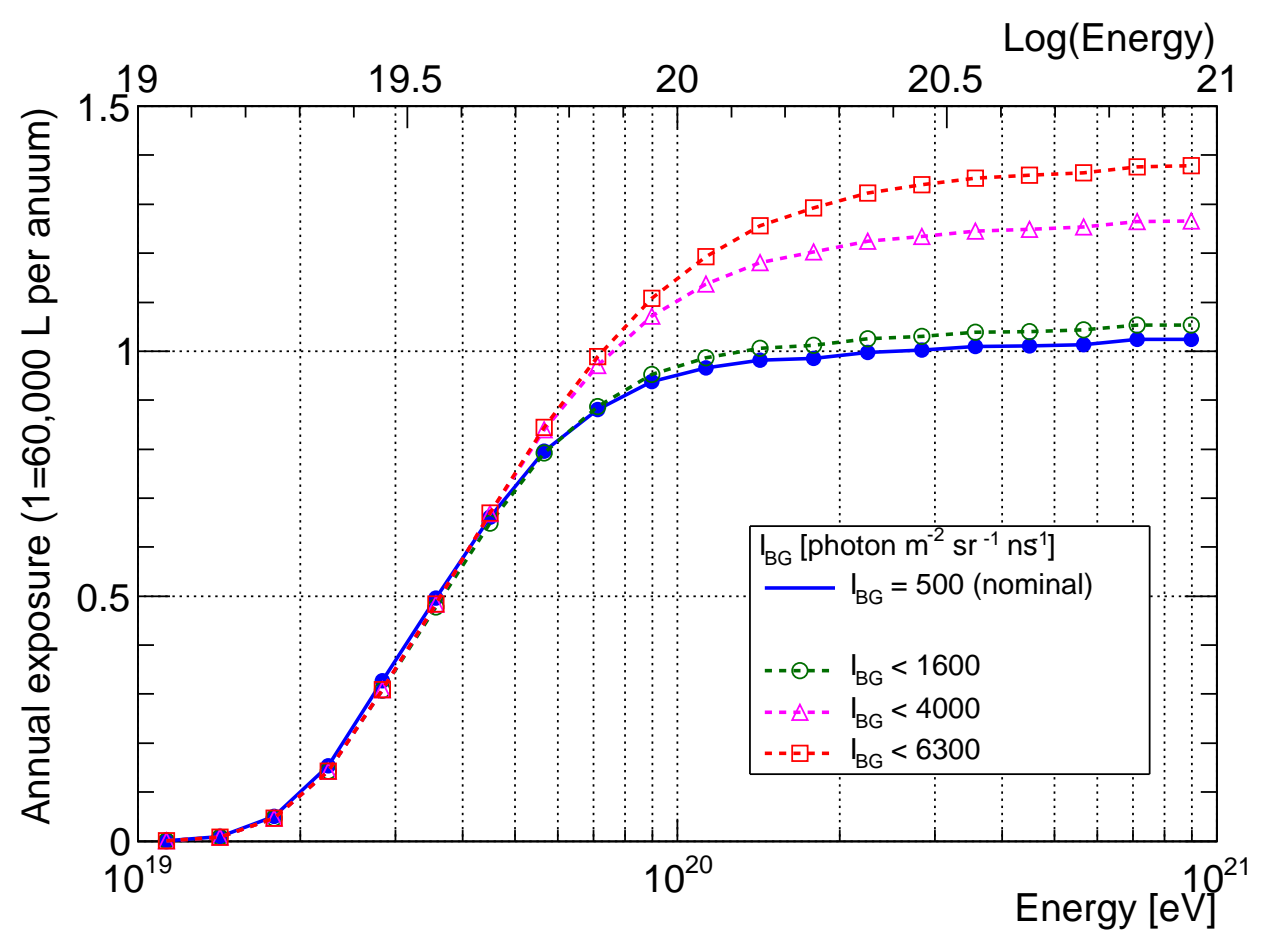

Figure 7: Exposure curve per year assuming the nominal background level of 500 photons $\mathrm{m}^{-2} \mathrm{sr}^{-1} \mathrm{~ns}^{-1}$ (blue curve). The unit is defined $60,000 \mathrm{~L}$ per annum $\left(1 \mathrm{~L}=1 \mathrm{~km}^{2} \mathrm{sr} \mathrm{yr}\right)$. The other curves indicate, in arbitrary units, the relative exposure to the nominal one for different values of maximum acceptable background level.

EAS or for the background, would be responsible to shift the energy scale of the aperture and exposure curves by the square root of the systematic uncertainty. On the other hand, the scaling factor would be linear in case it involves only the EAS propagation (i.e. hadronic interaction model and fluorescence yield) or if it is due to an error in the calculation of the attenuation of the UV signal.

\section{Signal discrimination from background}

The basic unit of information in the JEM-EUSO data stream is the so called photon-count, which is the number of photoelectrons counted by the pixels in $2.5 \mu \mathrm{s}$ or $1 \mathrm{GTU}$. In standard background conditions of 500 photons $\mathrm{m}^{-2} \mathrm{sr}^{-1} \mathrm{~ns}^{-1}$ the typical photon-count is $\sim 1$ count pixel ${ }^{-1} \mathrm{GTU}^{-1}$. Within the reconstruction scheme of ESAF, the photon-count information will be retrieved from the triggered PDMs and surrounding areas. This means that 
for each event we will have information of the photo-electron counts collected by every pixel of typically 8-9 PDMs (2304 channels per PDM) for the whole temporal extent of the event lasting a few hundreds $\mu$ s. On average, we deal with $2 \times 10^{6}$ photon counts per event, of which only a very small fraction, i.e. 500-5000 photon-counts depending on the EAS energy and arrival direction, are due to the actual EAS. The rest is diffuse background light. Therefore, a set of tools to disentangle the signal from the background has been developed, which has to be highly efficient to extract the $\sim 0.1 \%$ signal of the EAS from the total photon counts recorded in the event. These tools are called pattern recognition modules. For the sake of brevity, we will restrict to only two of the possible pattern recognition modules currently being developed or already available in ESAF: the Peak and WIndow SEarching (PWISE) technique and the LTT Pre-Clustering algorithm.

\subsection{Peak and WIndow SEarching (PWISE) technique}

The virtue of this module is that it selects photon-counts coming from the EAS, and at the same time it filters out multiple-scattered photons in the atmosphere and those that are not properly focused by the detector. These scattered photons produce a "fuzzy" image of the track. This effect appears as a consequence of their shifted arrival time due to the multiple scattering. The main goal behind the design of this module is to provide a clean track with enough photon-counts for the angular reconstruction algorithms, at the expenses of rejecting part of the signal of the event. We must always keep in mind that, due to the modularity of ESAF, we can use this pattern recognition module to assess the geometrical characteristics of the EAS. Afterward, we could select another module to reconstruct the energy once the geometry of the shower is known.

For the sake of clarity, we shall explain the PWISE technique step by step:

Step 1 For each pixel, PWISE only considers pixels whose highest photoncount (peak) is above a certain threshold (peak-threshold).

Step 2 Next PWISE searches for the time window with the highest signalto-noise ratio (SNR) defined by:

$$
\mathrm{SNR}=\left(\frac{1}{\Delta \tau \cdot \mathrm{RMS}}\right) \sum_{\Delta \tau} \operatorname{pc}(t)
$$

In this last expression $\mathrm{pc}(t)$ stands for the number of photon-counts as a function of time, $\Delta \tau$ is the time window's width centered on the 
aforesaid pixel's maximum peak, and RMS is the root-mean square of the pixel's photon-counts. Typically, $\Delta \tau$ takes values ranging between 1 and 7 GTUs, because the signal does not reside for longer times inside one pixel.

Step 3 We check if the maximum SNR is above a given SNR-threshold. Only if the SNR is above the threshold we select the photon-counts within the time window that maximizes SNR. The selected photoncounts are then passed on to the next reconstruction module.

In order to properly identify both, SNR-threshold and peak-threshold, events are simulated without background. This allows to view the SNR and peak of the photon-counts coming from the fluorescence and (scattered/reflected) Cherenkov light produced by the EAS. As mentioned before by selecting the appropriate thresholds, we select photon-counts that are produced by the EAS. Although, part of the photon-counts coming from the EAS are disregarded by the PWISE technique, among them the (multiple) scattered photons which are almost indistinguishable from the background and hence are prone to be filtered out.

\subsection{LTT Pre-Clustering}

The Linear Tracking Trigger (LTT) Pre-Clustering technique is an algorithm which can further enhance the performance of the angular reconstruction when applied preceding the actual pattern recognition. It is a refined version of the logic implemented in the $2^{\text {nd }}$ level trigger. It selects the pixels on the FS containing the highest number of counts. Then it searches for the track that maximizes counts by moving an integration box along a predefined set of directions intersecting this point. Pixels outside this track are ignored by the subsequent pattern recognition.

Figure 8 shows the $x$ - $t$ and $y$ - $t$ diagrams of the FS part containing the signal track for a shower with $10^{20} \mathrm{eV}$ from $60^{\circ}$ zenith angle after LTT PreClustering selection. Only a narrow track of pixels is selected to be handed over to the PWISE algorithm. The size of the box can be varied ad hoc. On average, almost independently of energy, by selecting a box with the size of an MAPMT $(8 \times 8$ pixels), more than $90 \%$ of the photon-counts originated by the EAS are retained by this procedure, with the advantage that the number of pixels, on which the PWISE algorithm is applied, is reduced to $\sim 3 \%$. 

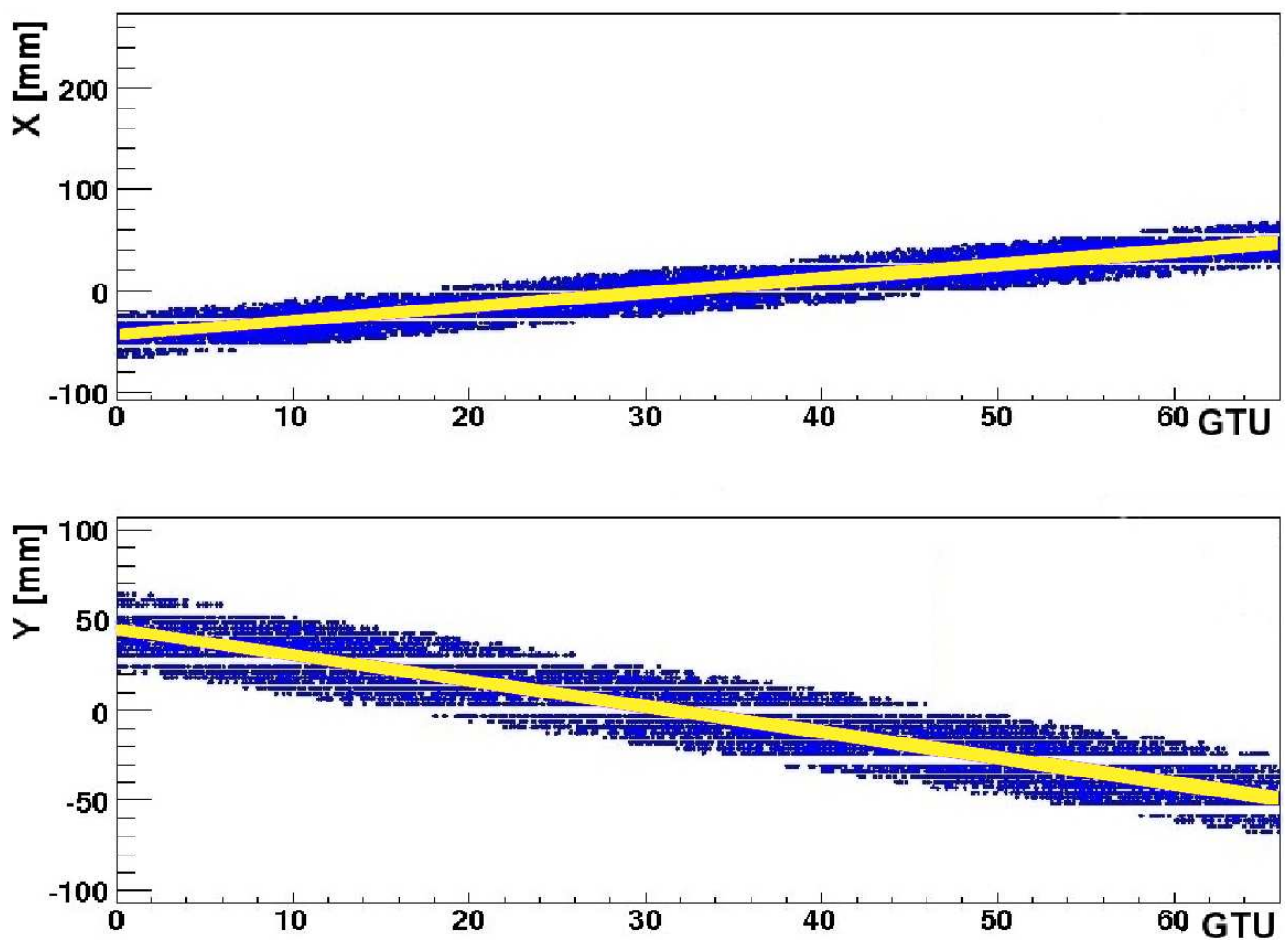

Figure 8: $x$ - $t$ (top panel) and $y$ - $t$ (bottom) diagrams of a $10^{20}-\mathrm{eV}$ shower $60^{\circ}$ zenith angle after application of the LTT Pre-Clustering algorithm. Blue points show the selected pixels with their position on the FS and timing on vertical and horizontal axes. The amount of pixels potentially containing the track being handed over to the PWISE algorithm is reduced significantly. The thick yellow line in the plots is an intermediate fit of the track and serves only as a guide for the eye.

\section{Reconstruction techniques to infer shower parameters}

The reconstruction techniques to infer shower parameters and the obtained results which are presented in this paper assume clear atmospheric conditions. The presence of clouds might complicate the reconstruction performance and in some cases might require specific reconstruction algorithms to improve the quality of results. These studies are currently on going and results will be reported in future. However, simulations in the past proved the feasibility of reconstructing EAS with reasonable uncertainty in the presence of clouds (Takahashi et al., 2009; Abu-Zayyad et al., 2004). 


\subsection{Arrival direction}

From the geometrical properties of the signal track on the FS the arrival direction of the primary can be computed by a variety of methods implemented in ESAF. Some algorithms are still being studied regarding their potential performance. Other methods are already well established and deliver results that fulfill the JEM-EUSO requirements in order to achieve the scientific objectives of the mission as explained in Section 1.

After signal discrimination the basic information to reconstruct the geometrical properties of the EAS is handed over to the track direction module. This is the signal track on the FS, i.e. the unique identifier of each pixel i, the number of detected photons for each pixel $N_{i}^{\text {hit }}$, their timing information $t_{i}$ and the viewing angle of the pixel $\hat{\mathrm{n}}_{i} . \hat{\mathrm{n}}_{i}$ is expressed by the incident an-

gle $\vartheta$ and azimuthal angle $\varphi$ of the incoming light to the optical axis of the telescope.

Then, we need to define the so-called Track-Detector-Plane (TDP) which is the plane containing the shower track and the detector itself. Figure 9 shows the system of the EAS and the detector. First step in angular reconstruction is the estimation of orientation of the TDP, by fitting the $x$ - $t$ and $y$ - $t$ histograms of the moving EAS image. From those fits the TDP position can be computed by two different kinds of algorithms, a numerical exact method and an analytical approximation (Pesce, 2004). After identification of the TDP a collection of different algorithms can be applied further to reconstruct the position of the air shower and hence the incoming direction of the primary is obtained. In the current configuration there are 5 different algorithms implemented in ESAF. Two of them yield the most promising performance:

Analytical Approximate 1 (AA1): Angular velocities $d \alpha / d t$ of the signal track in the $x$ - $t$ and $y$ - $t$ planes are derived from a linear fit. $\alpha_{i}$ denotes the angle between the direction of the light from the first data point identified in the track $\hat{\mathrm{n}}_{1}$ and the $i^{\text {th }}$ position $\hat{\mathrm{n}}_{i}$, hence $\alpha_{\max }$ between $\hat{\mathrm{n}}_{1}$ and $\hat{\mathrm{n}}_{\max }$. The shower maximum is identified from the maximum of a Gaussian fit on the count distribution in time. See Figure 10 for the geometrical relations.

The analytical relation between the angular velocity measured and the shower position is the following:

$$
\omega=\frac{d \alpha_{i}}{d t_{i}}=-\frac{c}{R_{\max }}\left[\frac{\sin ^{2}\left(\alpha_{i}+\beta\right)}{\sin \left(\alpha_{\max }+\beta\right) \cdot\left[\cos \left(\alpha_{i}+\beta\right)-1\right]}\right]
$$




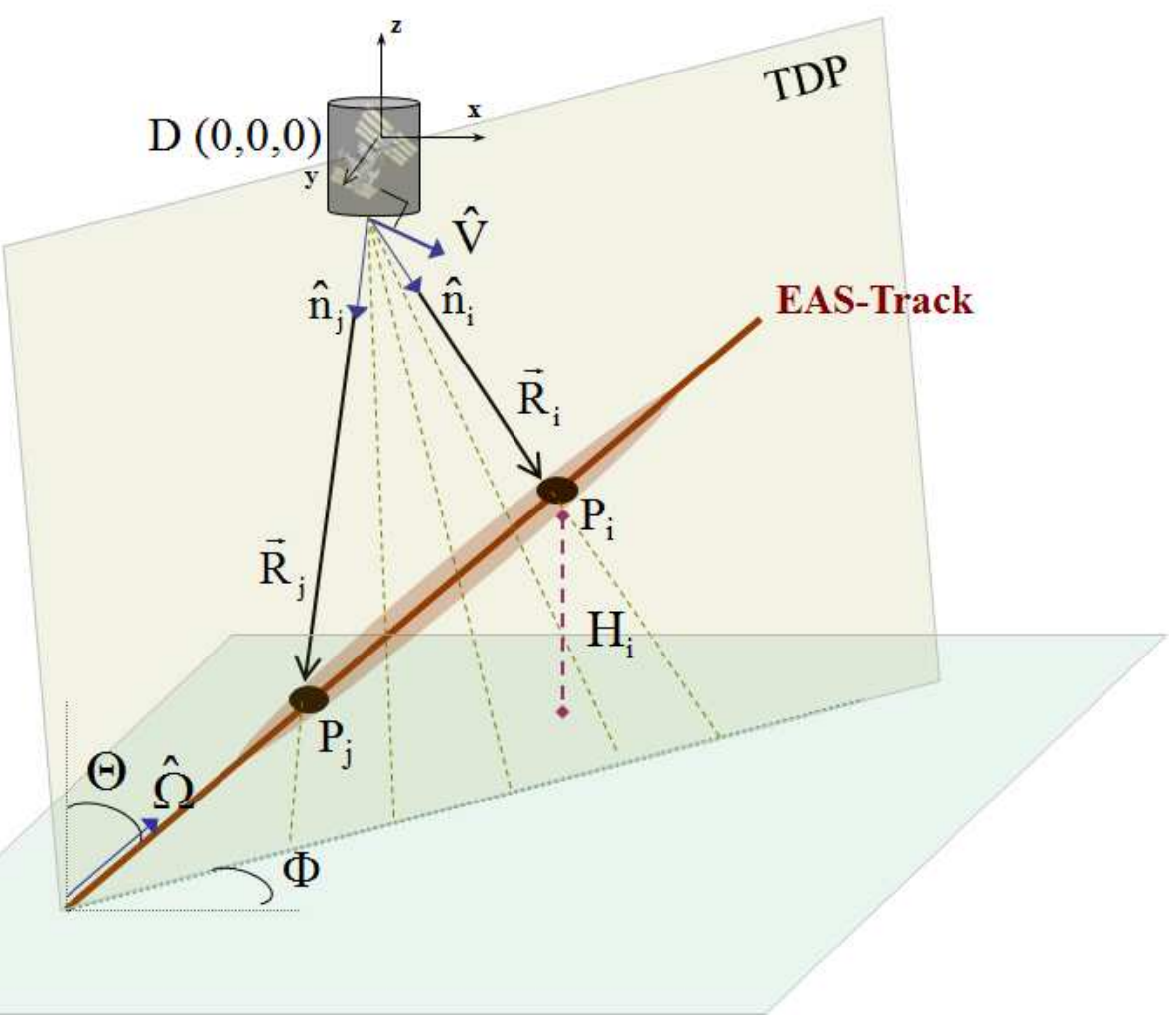

Figure 9: Illustration of an EAS observed by JEM-EUSO. On and near the track-detectorplane (TDP), photons emitted at times $t_{i}$ and $t_{j}$ reach the detector from certain directions $\hat{\mathrm{n}}_{i}$ and $\hat{n}_{j}$ after traversing $\overrightarrow{\mathrm{R}}_{i}, \overrightarrow{\mathrm{R}}_{j}$ in the atmosphere. From the timing information and incident direction of the shower photons, the arrival direction of the primary $\hat{\Omega}(\Theta, \Phi)$ is determined.

In the approximation $\alpha_{i} \approx \alpha_{\max }$ we get an expression for $\omega$ as a function of $\alpha_{\max }, R_{\max }$ and $\beta$ where $c=$ light velocity. For a detailed derivation see (Taddei et al., 2004).

The angular velocity $\omega$ is the slope of the line which results from the linear fit of $\alpha(\mathrm{t})$.

$$
\beta=2 \arctan \left(\frac{c}{\omega \cdot R_{\max }}\right)-\alpha_{\max }
$$

For the calculation of $\beta$ the distance $R_{\max }$ between the detector and the shower maximum is needed. As shown in (Pesce, 2004), it can be calculated by using $H_{\max }$, the altitude of the shower's fluorescence light maximum above 


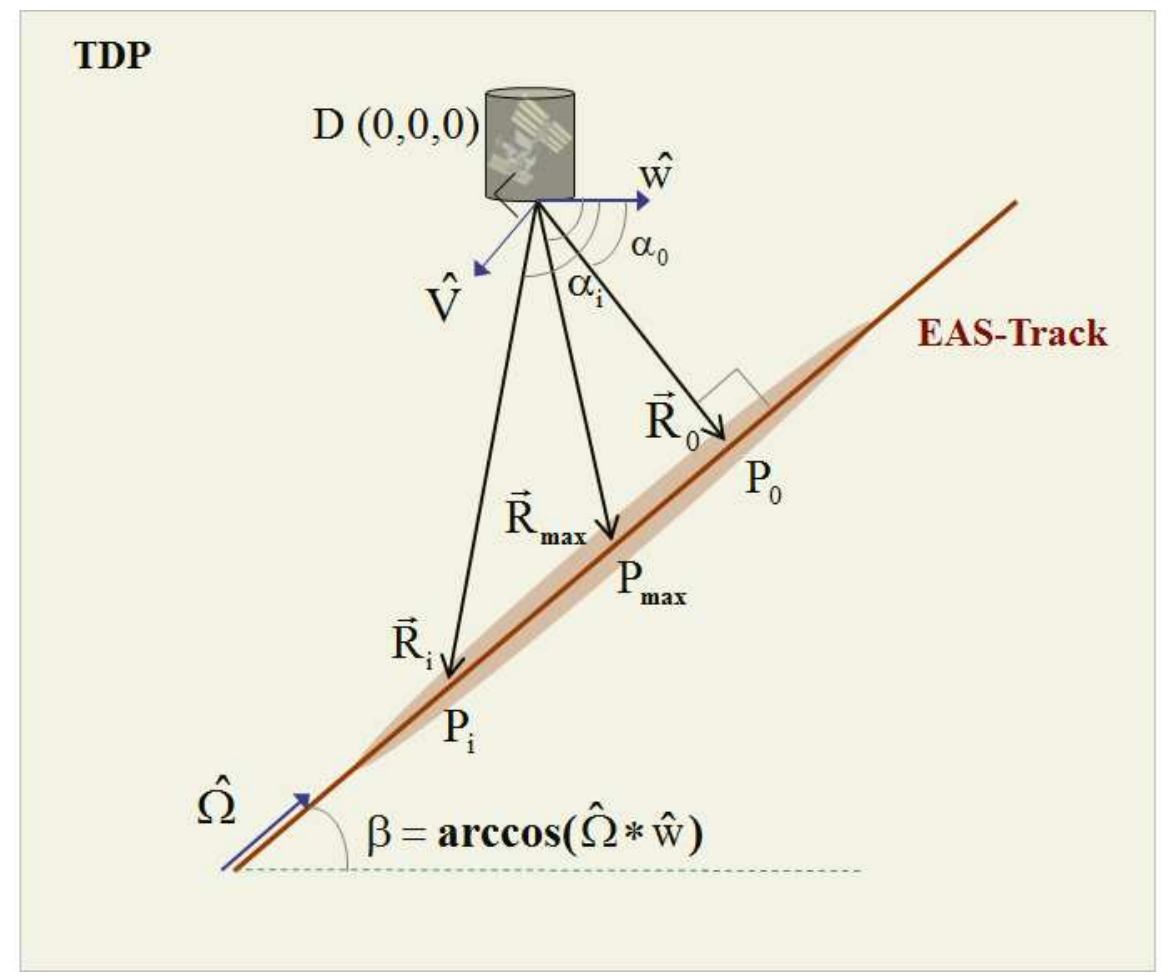

Figure 10: Side view of the track-detector-plane (see text for details).

the ground, and the unit vector $\hat{\mathrm{n}}_{\max }$ pointing to the maximum of the shower.

$R_{\max }=\left(R_{\oplus}+H_{\mathrm{ISS}}\right) \cdot \cos \vartheta_{\max }-\sqrt{\left(R_{\oplus}+H_{\max }\right)^{2}-\left(\left(R_{\oplus}+H_{\mathrm{ISS}}\right) \cdot \sin \vartheta_{\max }\right)^{2}}$

where $R_{\oplus}$ denotes the Earth's radius. In the formula $\vartheta_{\max }$ indicates the direction which the photons emitted around the shower maximum are coming from. Now, the arrival angle of the primary has to be derived in an iterative way. At first, an initial value for the expected $H_{\max }$ is assumed ad hoc, typically $5 \mathrm{~km}$. Together with the knowledge of the angular velocities of the photons reaching the instrument the shower position in TDP is computed. This result is used to recalculate $H_{\max }$. After recomputing this loop for some iterations the shower position is finally determined. The convergence probability of the algorithm depends on the shower conditions. For energies above $10^{20} \mathrm{eV}$ and $60^{\circ}$, usually more than $90 \%$ to $95 \%$ of the cases converge successfully.

Numerical Exact 2 method (NE2): This method does not require the con- 
cept of the TDP. Here, the reference is the last point of the signal track. This can be the position in which the air shower hits the ground or simply the Cherenkov point which indicates ground or cloud altitude. The altitude of this point has to be known. This limits the degrees of freedom (dof) to $\Theta$ and $\Phi$. We can now perform a $\chi^{2}$ minimization between the arrival angles of photons induced by the actual signal $\hat{\mathrm{n}}_{i}^{\text {measured }}$ to those induced by a test track $\hat{\mathrm{n}}_{i}^{\text {test }}$ (see Figure 11). For every pixel, the angle $\psi_{i}$, which is the angle between $\hat{\mathrm{n}}_{i}^{\text {measured }}$ and $\hat{\mathrm{n}}_{i}^{\text {test }}=\frac{\overrightarrow{\mathrm{R}}_{i}^{\text {test }}}{\left|\overrightarrow{\mathrm{R}}_{i}^{\text {test }}\right|}$, can be calculated.

$$
\psi_{i}(\Theta, \Phi)=\arccos \left(\hat{\mathrm{n}}_{i}^{\text {measured }} \cdot \frac{\overrightarrow{\mathrm{R}}_{i}^{\text {test }}}{\left|\overrightarrow{\mathrm{R}}_{i}^{\text {test }}\right|}\right)
$$

The $\chi^{2}$ function has a minimum once the test track matches the real one to a sufficient extent. Now, the missing parameters can be identified.

$$
\chi^{2}\left(\psi_{i}\right)=\sum_{\mathrm{i}=1}^{\mathrm{n}_{\text {pixel }}} \frac{\psi_{i}^{2}(\Theta, \Phi)}{\sigma_{i}^{2}} \cdot N_{i}^{\mathrm{hit}}
$$

The weight $N_{i}^{\text {hits }}$ is the number of photons hitting the $i^{\text {th }}$ pixel. $\sigma_{i}$ corresponds to the FoV of a single pixel. A detailed derivation of the relations can be found in (Bottai, 2003)

\subsection{Energy and Shower Maximum}

The PmtToShowerReco algorithm is the energy and $X_{\max }$ reconstruction algorithm developed in the framework of the JEM-EUSO mission. A detailed description of the algorithm and its performance can be found in (Fenu, 2013). In the following we summarize the key points. The algorithm works in an iterative way to better constrain the basic parameters of the shower. A very schematic view of the PmtToShowerReco algorithm is given in Figure 12.

The PmtToShowerReco receives as input the information on the timing and on the position for all the counts of a triggered event which is used to reconstruct the total signal intensity as a function of time (counts curve). All the time windows where the center of mass of the signal falls in proximity of a gap are removed (Fenu et al., 2011). The average atmospheric background level is subtracted in all the pixels selected in the track.

Following that, peaks in the reconstructed counts curve are searched to identify those from shower maximum and Cherenkov point. Such a search 


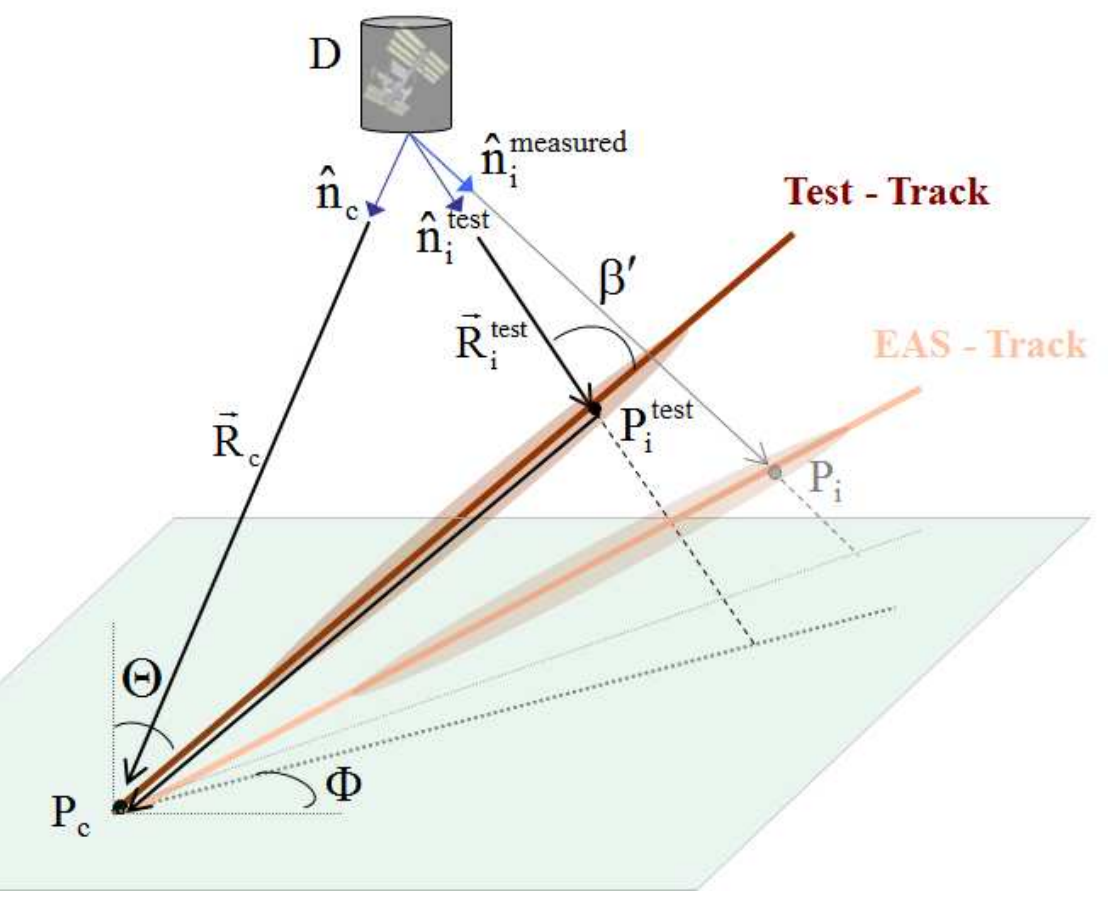

Figure 11: EAS observed with JEM-EUSO: Photons emitted by the shower reach the detector from certain directions $\hat{\mathrm{n}}_{i}, \hat{\mathrm{n}}_{j}$ after traversing $\mathrm{R}_{i}, \mathrm{R}_{j}$ in the atmosphere. The comparisons between the incident directions of the shower photons to those of a test EAS from different arrival directions allow to reconstruct $\hat{\Omega}(\Theta, \Phi)$.

is done on the counts curve but it can be equally done on the photons at the pupil level. The difference between the two cases is anyway minimal. The peak search is done by a devoted algorithm developed on simulated events. It copes with the quite discontinuous and irregular nature of the signal, and the gaps that cause sudden decreases in the light intensity, which can deplete the counts curve. The Cherenkov-peak identification depends on energy and angle. It is higher than $70 \%$ for energies $E>5 \times 10^{19} \mathrm{eV}$ and $\Theta<45^{\circ}$, and grows with energy still remaining higher than $80 \%$ for energies $E>3 \times 10^{20}$ and $\Theta<60^{\circ}$. The time separation between the peaks is then used to reconstruct the geometry of the shower. Considering the propagation of the photons in atmosphere and the light-speed of the shower, the distance between maximum and ground is calculated. This defines the position of the 
maximum since the altitude of the ground is known.

The number of detected photo-electrons as a function of time is converted into the number of photons hitting on the FS, by taking into account the efficiency of the MAPMTs, the loss of the FEE and the transmittance of the optical filter.

Following that, the correction of the optics effects is performed. The final output of such a procedure consists in the light curve at the entrance pupil. Within ESAF the so called OpticsResponse is calculated to estimate the optics efficiency. By means of an independent algorithm in ESAF, several point sources are simulated following a set of predefined directions in $\vartheta$ and $\varphi$ of the incident photon. All the corresponding photons are also simulated according to a predefined set of wavelengths (from 250 to $500 \mathrm{~nm}$ in 25-nm steps). Given a certain number of photons, several factors are measured. The first one, named Optics Efficiency, is the ratio of photons reaching the FS to those incident on the telescope pupil and is $\sim 40 \%$ for $\vartheta=0^{\circ}$ and $\sim 20 \%$ at $30^{\circ}$. The second factor, named Spot Collection Efficiency, expresses the ratio between the photons collected inside a predefined radius of $3 \mathrm{~mm}$ with those reaching the FS. The latter is more relevant since it expresses the capability of focusing signal inside a limited area that enhances the signalto-noise ratio. Around the wavelength band of interest, the convolution of the two efficiencies is $\sim 35 \%$ for $\vartheta=0^{\circ}$ and is $\sim 15 \%$ at $30^{\circ}$, comparable to Table 1.

Moreover, the Pixel-Angle-Map algorithm maps the arrival direction of the photons depending on the pixel under analysis. After calculating the average incident direction of all the generated photons for each time, it is possible to retrieve from the map an average optics efficiency which is the factor used for the calculation of the photon curve at the optics entrance. For each GTU the number of photons on the FS are divided by this efficiency, delivering the amount of photons on pupil.

The estimation of the luminosity of the shower (the module photons in shower in Figure 12) follows next. The $1 / R^{2}$ flux-decrease factor due to the shower distance from the detector can be accounted for just by projecting on the Earth the average spot angular direction. This procedure is affected by a minimal systematics since the shower is developing in the lowest $20 \mathrm{~km}$ while JEM-EUSO is placed $400 \mathrm{~km}$ above the Earth's surface. On the other hand, a precise knowledge of the position in the atmosphere is needed for the atmospheric transmission correction. Due to the monocular nature of the JEM-EUSO mission, that does not allow the direct observation of the radial 


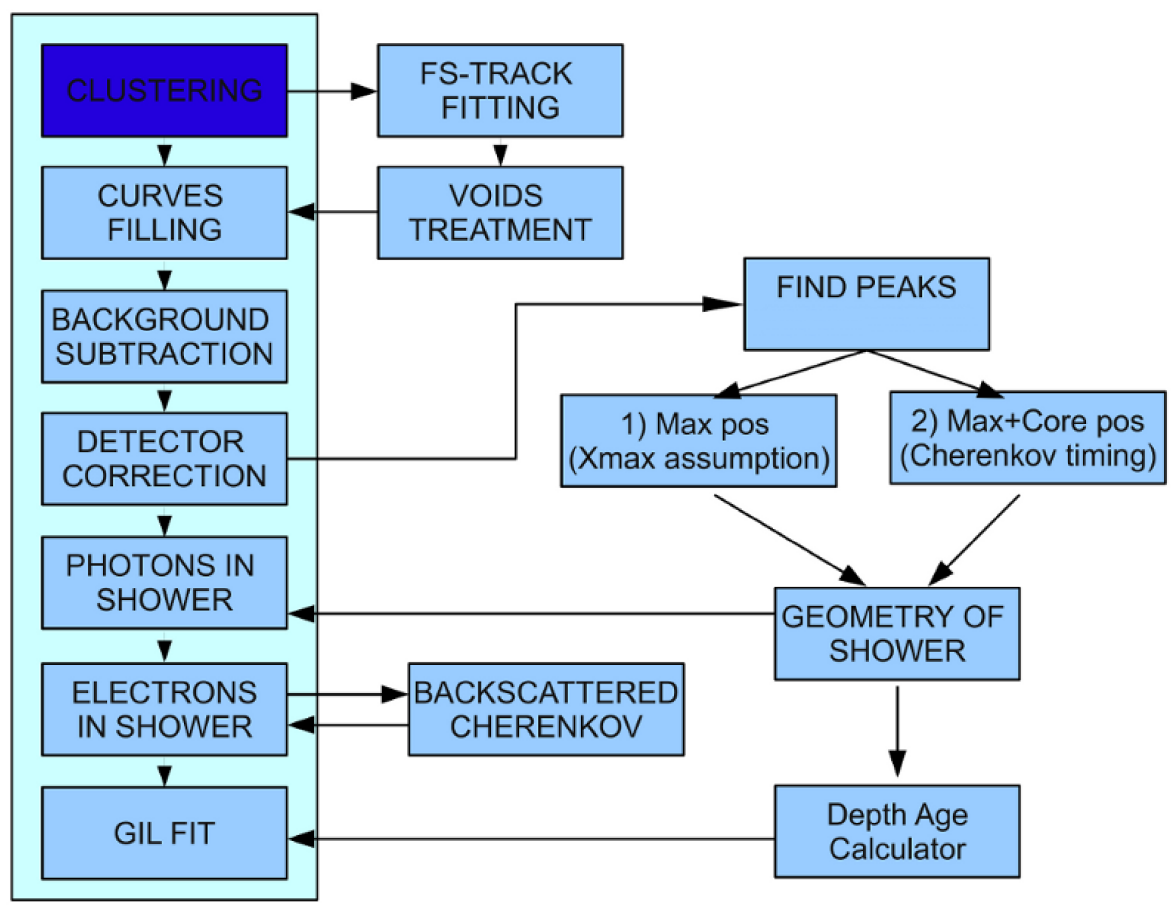

Figure 12: Schematic view of the PmtToShowerReco algorithm. The present scheme represents the operations performed on the event curves on the left. On the right part of the plot the complementary operations are shown. More in detail the left sequence of boxes represents from top to bottom: the track reconstructed by the pattern recognition algorithms, the filling of the counts curve, the correction of the background (here the counts curve is obtained), the electronics and optics correction (here the light curve is obtained), the distance and absorption correction, the calculation of the electron curve and finally the fit. On the right side we see the correction (or removal of the gaps), the recognition of the Cherenkov-peak and shower maximum, the geometrical reconstruction as well as the back-scattered Cherenkov light correction.

motion of the shower, devoted algorithms are needed to assess the position of the shower inside the atmosphere.

Two methods have been developed for the altitude determination. The first makes use of the Cherenkov point while the second assumes a parametrization for the depth of $X_{\max }$ and relies on the direction delivered by the algorithms for direction reconstruction.

The first method, referred to as 'Cherenkov method', depicted in Figure 13 assumes the identification of a Cherenkov point within the counts curve. 

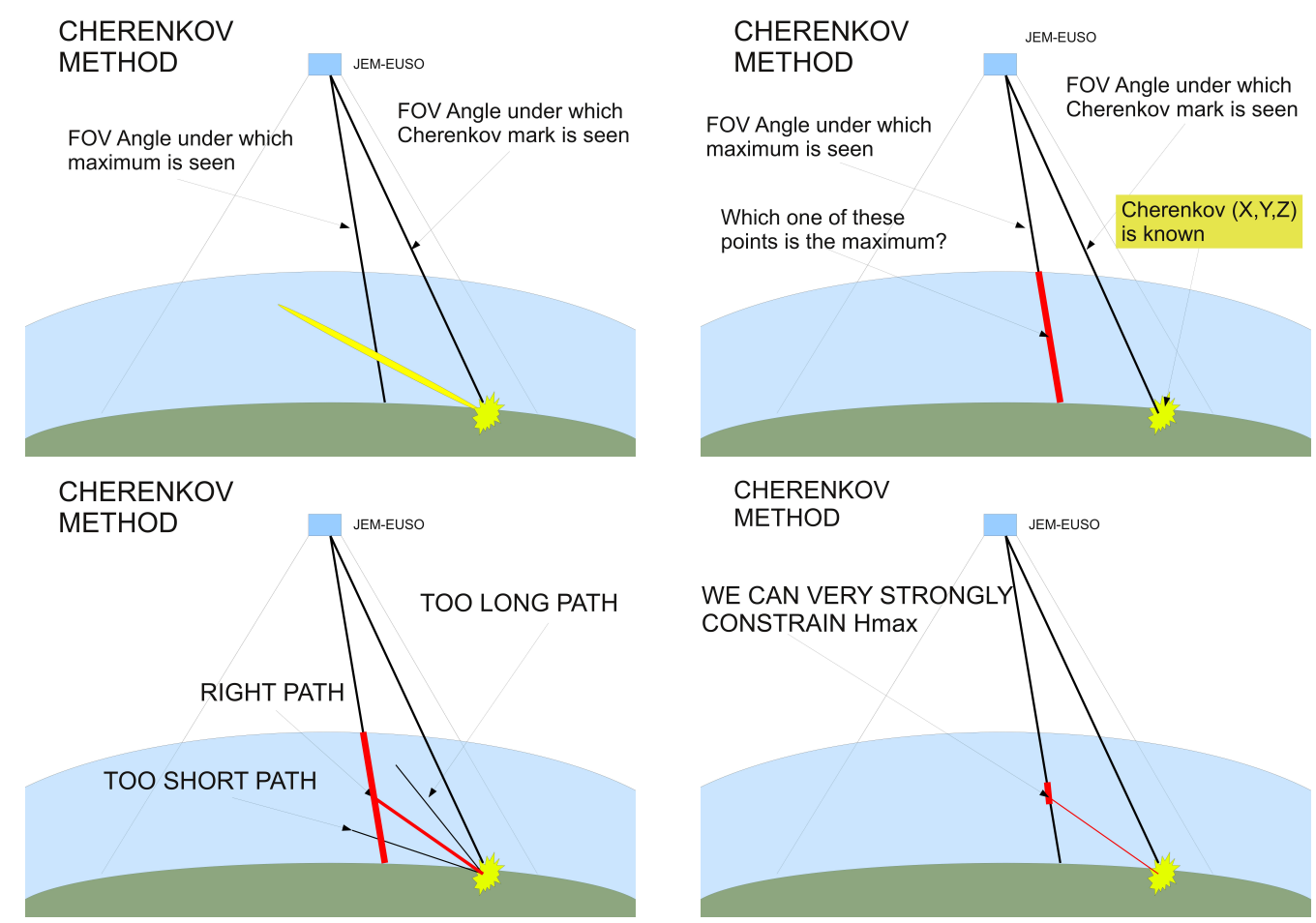

Figure 13: First method, referred to as 'Cherenkov method', used to determine the altitude of the shower maximum which makes use of the Cherenkov point at Earth's surface (topleft). Without knowing the time and position of the Cherenkov mark, any position inside the red line in top-right plot and bottom-left plot could be in principle the location of the shower maximum. However, if the time and position of the Cherenkov point is known, only a limited range of altitudes for the shower maximum becomes compatible with both the location of the Cherenkov point and of the timing and FoV of the pixels where the maximum intensity of the shower is identified (bottom-right).

The Cherenkov peak identification is quite a difficult task which requires a devoted algorithm. As a first task, a preliminary selection of the peaks in the count curve must be performed. The definition of peak assumed in this work requires that the peak is the local maximum within the \pm 2 GTUs window centered around it. Following that, an analysis ordering such local peaks from the higher to the lower must be performed together with an analysis on the timing and on the position. Peaks near the gaps must be treated in a devoted way since the probability for them to be local maxima is higher than in the other cases. Moreover peaks not satisfying criteria of a good signal to noise ratio or isolated peaks must be rejected. In such a way the long tail of small 
isolated peaks in the later stages of inclined showers is suppressed. Generally the highest peak is assumed to be the shower maximum. The Cherenkovpeak is recognized as the last significant peak in the time development of the shower. An additional possibility is using a parametrization of a shower development from a hadronic primary for validating the time delay between the maximum and the Cherenkov-peak. In fact assuming a particular type of primary forces the interval between the light intensity of maximum and the Cherenkov-peak to be within a certain interval. Generally with increase of zenith angle, Cherenkov light is more attenuated and cone-like Cherenkov emission makes the reflection pattern wider on the Earth's surface. This results in a worse signal-to-noise ratio of the Cherenkov-peak. Therefore, this method allows the peak recognition up to $\sim 60^{\circ}$ in zenith angle. Moreover gaps are to be considered the likely cause of a $15 \%$ loss of efficiency below such angles. For this reason the Cherenkov method is not always applicable and tends to fail for the most inclined showers.

Once the Cherenkov-peak is identified, the time delay between shower maximum and Cherenkov point delivers the distance traveled by the shower particle. Taking into account the direction of the pixels in which the shower maximum and the Cherenkov-point are detected, the altitude of the shower maximum is inferred. This process is achieved through a procedure sampling several geometries in the FoV. Given a timing separation between two directions of light intensity, maximum and Cherenkov point, from JEM-EUSO, the maximum will be chosen to be the one satisfying the timing constraint. Obviously a proper calculation must take into account both shower and photons propagation times.

The second method, referred to as 'No-Cherenkov method', is depicted in Figure 14. This method assumes instead that a direction reconstruction has been already concluded successfully and that the maximum of the shower is identified and its projection on the FoV constrained. At this point there is only a limited range of altitudes of shower maximum that can comply with the above constraints according to a specific primary particle and a chosen parametrization of the shower development (GIL in our case but any other $X_{\max }$ parametrization can be chosen as well). The ranges of shower maximum in depth and altitude are large enough to well contain the fluctuations of shower maxima for both proton and iron primaries.

Once the three-dimensional position of the maximum is determined, the position of the shower at each time is calculated, as well as a first estimation of parameters like slant depth of atmosphere and age parameter for each step 


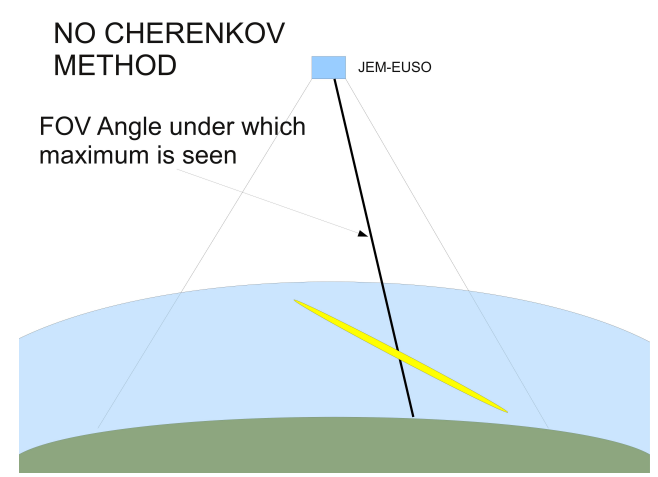

We already reconstructed

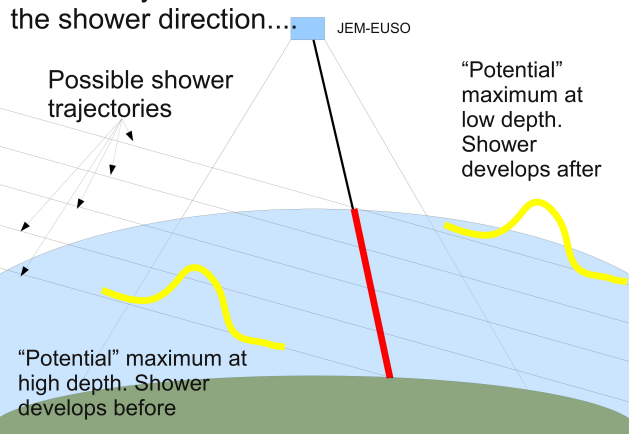

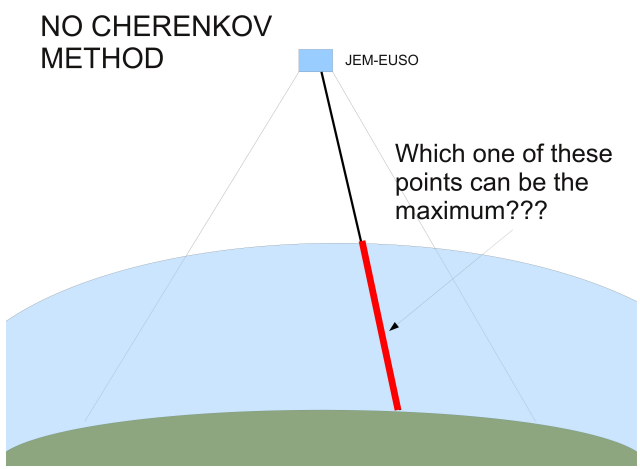

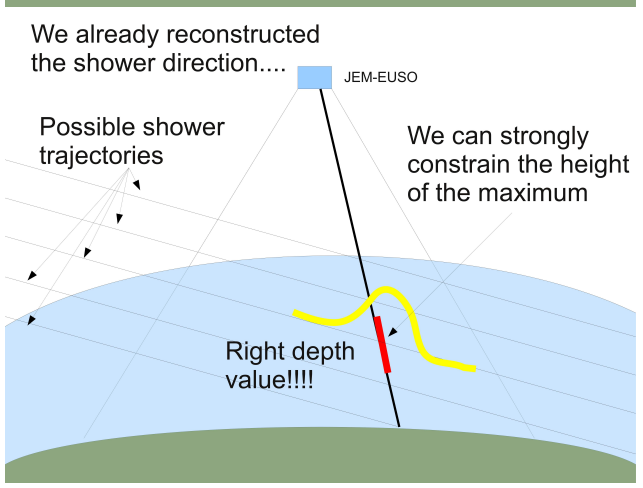

Figure 14: The second method, referred to as 'no Cherenkov method', to determine the altitude of the shower maximum assumes the knowledge of the shower direction (top-left). There is only a limited range (bottom-right) of the possible altitudes (top-right) compliant with the assigned direction of the shower, and the timing and FoV of the pixels where the maximum intensity is observed. In the rest of the cases the shower profile is not compliant (bottom-left).

of the shower track.

With all these information, the luminosity of the shower is calculated. The modeling of the transmittance in US 1976 Standard Atmosphere (NOAA et al., 1976) is assumed as in LOWTRAN (Kneizys et al., 1988) software. Details can be found in (Fenu, 2013). Such a software is used both in simulation and in reconstruction, therefore, the uncertainty in the geometrical reconstruction is the only systematic effect affecting the amount of transmitted light. At the end of this process the number of photons for each time and as a function of the slant depth is obtained.

Unfortunately no distinction is possible on whether the detected photons are originated by fluorescence or Cherenkov emissions. Moreover, the knowledge of the number of electrons is necessary in order to estimate the pro- 
duction of Cherenkov photons. For this reason an iterative process has been developed in order to calculate the Cherenkov light contamination. The first shower step is assumed to be Cherenkov-light-free in order to perform a first calculation of the electron curve and of the contribution from Cherenkov light. The fluorescence yield as derived in (Nagano et al., 2004) is applied to convert the photon number into an electron number. The energy distribution modeled by (Giller et al., 2004) is applied to the electrons. At this point a certain amount of Cherenkov photons are produced and carried out to the next step and partially scattered according to the LOWTRAN parametrization. In the present calculation the asymmetric distribution of the Rayleigh scattering and the ozone component are also taken into account. The scattered component is, therefore, assigned to the following step and set equal to the contamination. The surviving component (neither scattered nor absorbed) is delivered to the next step. The process starts again from the following step after subtracting the contamination fraction to the total amount of reconstructed photons. The new shower's electron number is used for the Cherenkov light production calculation. The amount of produced Cherenkov light is summed to the one inherited from the previous steps. The total amount is propagated and the fraction of scattered photons assigned as contamination to the next step. The process repeats to the end of the shower till a contamination value is retrieved for each shower's step along the track.

At this stage the final electron curve is obtained and fitted by a GIL function (but any other function is equally applicable) in order to obtain the energy and a more reliable value for the $X_{\max }$ parameter. The entire procedure is repeated by applying an iterative variation of the shower's parameters and a simulation in order to define the set of parameters that best fits to the reconstructed detector response. It has to be reminded that in some cases the location of the signal maximum does not correspond to the location of the shower maximum. The latter is obtained by applying the corrections to the event and reconstructing the shower profile. Therefore, errors in the reconstruction of the shower direction will affect also the reconstruction of the shower maximum and the energy estimation. For this reason an iterative procedure is applied in order to minimize such uncertainties. The results presented in this paper and their uncertainties take into account also this possible source of error. 
Another way of describing the process of Figure 12 is shown as follows:

$$
\begin{aligned}
& \frac{d N_{\mathrm{pe}}}{d L}=\int_{\lambda_{\min }}^{\lambda_{\max }}\left\{N_{\mathrm{e}}\left(X(L), X_{\mathrm{max}}, E\right) \cdot \frac{d \epsilon_{\mathrm{F}}(H(L), s(L))}{d \lambda} \cdot \frac{1}{4 \pi}\right. \\
& +\int_{0}^{L}\left\langle N_{\mathrm{e}}\left(X(l), X_{\max }, E\right) \cdot \frac{\epsilon_{\check{\mathrm{C}}}(H(l), s(l))}{C^{\prime} \lambda^{2}}\right. \\
& \cdot \exp \left[-\tau_{\mathrm{s}}(\lambda, \overrightarrow{\mathrm{P}}(l), \overrightarrow{\mathrm{P}}(L))-\tau_{\mathrm{a}}(\lambda, \overrightarrow{\mathrm{P}}(l), \overrightarrow{\mathrm{P}}(L)]\right\rangle d l \\
& \cdot\left\langle 1-\exp \left[-\tau_{\mathrm{s}}\left(\lambda, \overrightarrow{\mathrm{P}}\left(L-\frac{d L}{2}\right), \overrightarrow{\mathrm{P}}\left(L+\frac{d L}{2}\right)\right)\right]\right\rangle \cdot f^{\prime}(\tilde{\beta}(\overrightarrow{\mathrm{P}}(L), \vec{\Omega})\} \\
& \cdot \exp \left[-\tau_{\mathrm{s}}(\lambda, \overrightarrow{\mathrm{P}}(L), \overrightarrow{\mathrm{D}})-\tau_{\mathrm{a}}(\lambda, \overrightarrow{\mathrm{P}}(L), \overrightarrow{\mathrm{D}})\right] \cdot \frac{A_{\mathrm{opt}} \cdot \cos \vartheta}{R^{2}} \\
& \cdot \varepsilon_{\mathrm{opt}}(\lambda, \vartheta, \varphi) \cdot T_{\mathrm{BG} 3}(\lambda) \cdot \varepsilon_{\mathrm{PMT}}(\lambda) \cdot \varepsilon_{\mathrm{FE}} d \lambda
\end{aligned}
$$

This equation shows the amount of counts $d N_{\text {pe }}$ detected by JEM-EUSO at $\vec{D}$ from line element $d L$ along the EAS track at the position $L . X(L)$ and $H(L)$ represent the slant depth along the EAS and the altitude from the Earth's surface, respectively. First of all, $d N_{\mathrm{pe}}$ depends on the number of electrons in the shower $N_{\mathrm{e}}$ that on its own is parametrized by $E$ and $X_{\max }$ and is a function of $X$. Another fundamental factor is the fluorescence yield $\epsilon_{\mathrm{F}}$, which is a function of $H$ and the age parameter $s$ of the EAS. $s$ is a function of $X$ that consequentially determines the energy deposit by all the electrons in the EAS. Here $\epsilon_{\mathrm{F}}$, is shown in the differential of wavelength $d \lambda$ to represent the characteristic spectrum. These fluorescence photons are isotropically emitted in $4 \pi$ sr solid angle.

In addition Cherenkov photons may contribute to the detected counts. The amount of Cherenkov photons produced is also proportional to $N_{\mathrm{e}}$ and the Cherenkov yield $\epsilon_{\check{\mathrm{C}}}$, i.e. number of Cherenkov photons emitted per traversed distance of electron. As Cherenkov light has a characteristic $\lambda^{-2}$ spectrum, $\epsilon_{\check{C}}$ is meant in the band between $\lambda_{\min }$ and $\lambda_{\max } \cdot C^{\prime}=\lambda_{\min }{ }^{-1}-\lambda_{\max }{ }^{-1}$ is a normalization constant for the yield. However, those photons are beamed nearly parallel to the EAS track and only those scattered at $L \pm d L / 2$ and towards the JEM-EUSO telescope might be detected. Before reaching the position $L$, a part of Cherenkov photons produced at $l$ may be extinguished. This extinction process is written by $\exp \left[-\tau_{\mathrm{S}}-\tau_{\mathrm{A}}\right]$ where $\tau_{\mathrm{S}}$ and $\tau_{\mathrm{A}}$ are scattering and absorption optical depths, respectively. In order for Cherenkov photons to be observed by JEM-EUSO in direction of position $L$, such photons need to be scattered towards JEM-EUSO. Similarly, the probability of 
photons scattered at $L \pm d L / 2$ is presented as $1-\exp \left[-\tau_{\mathrm{S}}\right]$ as seen in the third line of Equation (10). $f^{\prime}$ is the phase function to give the probability per solid angle that photons are scattered to the JEM-EUSO direction at $\tilde{\beta}$ angle between direction of the shower track and line of sight at $L$. The summation of the two light components is the group of photons moving towards the JEM-EUSO telescope from the position $L$. These photons also suffer from scattering on the way to the telescope with transmittance $\exp \left[-\tau_{\mathrm{S}}-\tau_{\mathrm{A}}\right]$. After taking into account these factors, only the photons moving within the solid angle of the telescope pupil $A_{\text {opt }} \cdot \cos \vartheta / R^{2}$ may be seen where $\vartheta$ is the viewing angle of the line of sight and $R$ is the distance to telescope from position $L$.

In the telescope, the arrival photons are transferred with known response functions of optics (photon collection efficiency), MAPMT (i.e. detection efficiency), BG3 filter (transmittance) and FEE, that are given by $\varepsilon_{\text {opt }}(\lambda, \vartheta, \varphi)$, $T_{\mathrm{BG} 3}(\lambda), \varepsilon_{\mathrm{MAPMT}}(\lambda)$, and $\varepsilon_{\mathrm{FE}}$, respectively. These instruments are optimized to the wavelength band for fluorescence light detection. The integration is performed over a wider band, between $\lambda_{\min }=250 \mathrm{~nm}$ and $\lambda_{\max }=485 \mathrm{~nm}$ where the efficiency is practically zero, in order to have a signal count $d N_{\text {pe }}$ from the element $d L$ comparable to the observed data.

As seen in Equation (10), most of the factors are wavelength dependent. The effective range of $\lambda$ is determined by the transmittance of BG3 filter and optics response and it is between $\sim 300 \mathrm{~nm}$ and $\sim 430 \mathrm{~nm}$. The scattering optical depth in the atmosphere is dominated by Rayleigh scattering and is proportional to $\lambda^{-4}$. Concerning absorption, it is only dominant in shorter wavelengths $\lambda \lesssim 320 \mathrm{~nm}$ due to ozone absorption.

\section{Reconstruction results in clear-atmosphere conditions}

\subsection{Direction Reconstruction}

As a first approach to asses ESAF's current angular reconstruction capability, we simulate a set of proton initiated EAS and then compared the angle between the simulated and reconstructed arrival directions. We name $\gamma$ the angle between the simulated and the reconstructed arrival directions. In order to express the error of arrival direction, taking the probability density function $p(\gamma)$ of this angle between $\gamma$ and $\gamma+d \gamma$, we define $\gamma_{68}$ as:

$$
\int_{0}^{\gamma_{68}} p(\gamma) d \gamma=0.68 \int_{0}^{\pi} p(\gamma) d \gamma
$$



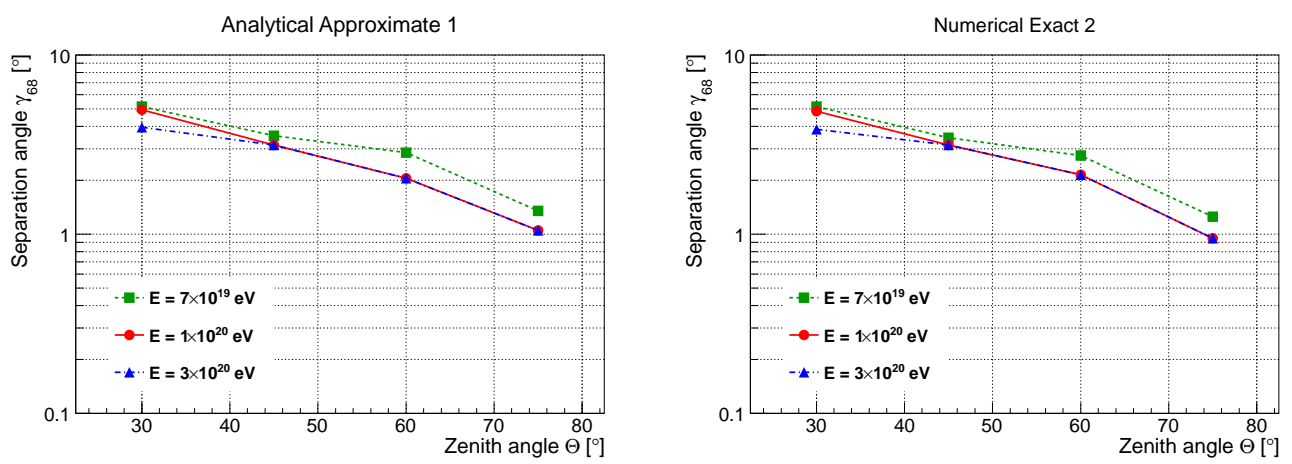

Figure 15: Preliminary results for JEM-EUSO's angular reconstruction error $\gamma_{68}$ as a function of the zenith angle using the AA1 (left) and NE2 (right) algorithms (see text for details).

$\gamma_{68}$ represents the minimum value that fulfills that $68 \%$ of $\gamma^{\prime}$ 's are less than $\gamma_{68}$. This parameter is used as a measurement of the overall performance of reconstruction capabilities. It is worth mentioning that both systematic errors and statistical fluctuations are included in the definition of $\gamma_{68}$.

For this work we simulate a set of EASs which consist of EASs with energies of $7 \times 10^{19} \mathrm{eV}, 10^{20} \mathrm{eV}$, and $3 \times 10^{20} \mathrm{eV}$. For each one of these energies we simulate $\approx 1000$ EASs at four fixed zenith angles, namely $\Theta=30^{\circ}, 45^{\circ}$, $60^{\circ}$, and $75^{\circ}$; while the azimuth is randomly taken between $0^{\circ}$ and $360^{\circ}$. The results of this preliminary study for both AA1 and NE2 algorithms are shown in Figure 15. The standard background conditions of 500 photons $\mathrm{m}^{-2} \mathrm{sr}^{-1} \mathrm{~ns}^{-1}$ are assumed, which correspond on average to $\sim 1$ count pixel ${ }^{-1}$ $\mathrm{GTU}^{-1}$.

Since JEM-EUSO is observing the EAS's track from above, the more vertical the EAS is, the harder it will be to reconstruct it (e.g. $\Theta \leq 45^{\circ}$ ). This complication arises since these EASs are seen by relatively less pixels than EAS more parallel to the Earth's surface for large zenith angle showers.

While there is still room to improve, we can conclude that the present preliminary status already satisfies the scientific requirements (Bertaina et al., 2011) of the mission. We currently investigate more refined selection mechanisms and the same goes for angular reconstruction algorithms. We are confident that in the nearby future, we will improve these results. As an extra outcome of the study presented here, we have tested the robustness of the ESAF framework for simulating space borne detectors. 


\subsection{Energy Reconstruction}

As an example to show the performance of the energy reconstruction, the result of a simulation of a set of 250 proton events extracted randomly in a squared region of $200 \mathrm{~km} \times 200 \mathrm{~km}$ centered around the nadir location with energy $E=10^{20} \mathrm{eV}$ and zenith angle variable between $20^{\circ}$ and $70^{\circ}$ is presented in the following. The nominal background level of 500 photons $\mathrm{m}^{-2} \mathrm{sr}^{-1} \mathrm{~ns}^{-1}$ is assumed, and the 'No-Cherenkov method' is applied to the energy reconstruction. Left side of Figure 16 shows an example of a typical reconstructed shower profile together with the simulated one. The reconstructed energy is $E_{\text {rec }}=9.74 \times 10^{19} \mathrm{eV}$, while $\Theta_{\text {rec }}=61.2^{\circ}$ for an input $\Theta_{\text {true }}$ of $60.2^{\circ}$ ). The reconstructed shower profile fitting the data has a reduced chi square $\chi^{2} /$ dof $=0.51$. Of the 250 events simulated, 25 are discarded because of high reduced $\chi^{2}$ values $\left(\chi^{2} /\right.$ dof $\left.>3\right)$ or because the event reconstruction does not converge on a reasonable fit. Right side of Figure 16 shows the distribution of the reconstructed energies. The determination error of energy is $\Delta E / E \sim 18 \%$ at $68 \%$ confidence level, with a slight systematic effect of $\sim 10 \%$. The origin of this offset is under investigation and this is part of the optimizations that are currently going on. The average value of the reduced $\chi^{2} /$ dof is 1.02 . Regarding the equivalent $X_{\max }$, the determination error is $\Delta X_{\max } \sim 110 \mathrm{~g} \mathrm{~cm}^{-2}$. However, if events above $45^{\circ}$ are considered the resolution improves to $\Delta X_{\max } \sim 85 \mathrm{~g} \mathrm{~cm}^{-2}$. This is due to the fact that the longer tracks simplify the reconstruction of the shower profile. A better angular reconstruction allows a better identification of the maximum of the shower, and therefore, $X_{\max }$.

\section{Conclusions}

The scientific requirements of the JEM-EUSO mission related to the analyses presented in this paper are the following: Observation area greater than $1.3 \times 10^{5} \mathrm{~km}^{2}$; Arrival direction determination accuracy better than $2.5^{\circ}$ for $E>10^{20} \mathrm{eV}$ and $\Theta=60^{\circ}$ (standard showers ${ }^{6}$ ); Energy determination accuracy better than $30 \%$ for standard showers; $\Delta X_{\max }<120 \mathrm{~g} / \mathrm{cm}^{2}$ (JEM-EUSO Collaboration, 2010). Simulations show that JEM-EUSO reaches almost full efficiency already at energies around $3 \times 10^{19} \mathrm{eV}$ for a restricted subset of

\footnotetext{
${ }^{6}$ As standard shower the authors indicate a $10^{20}-\mathrm{eV}$ proton initiated shower with a zenith angle of $60^{\circ}$.
} 

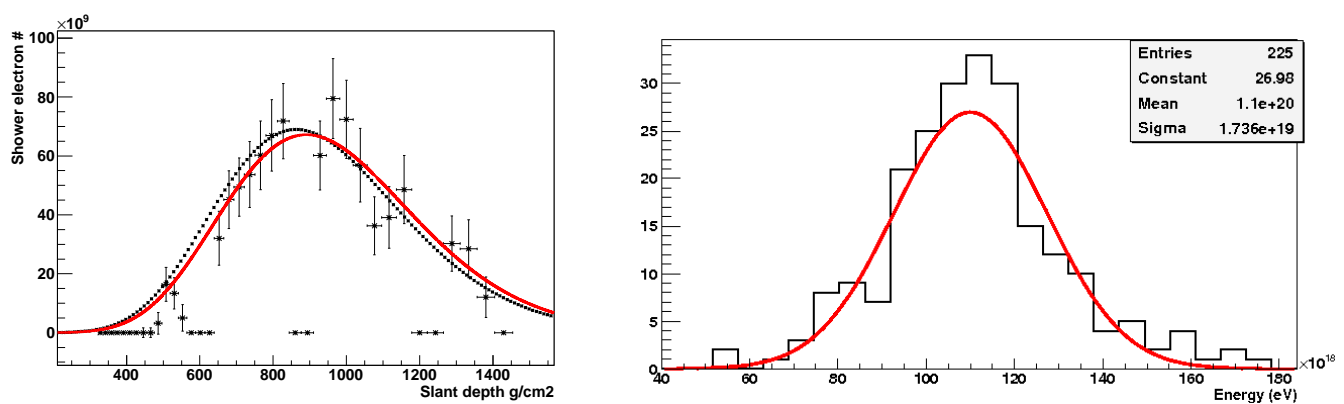

Figure 16: Left panel: Reconstructed shower profile of a typical simulated event. Data points indicate the reconstructed electron number as a function of depth after all instrument and atmospheric corrections have been applied. Black dashed curve shows the original profile, while the red line the reconstructed one from the fit of the data points. Right panel: Energy distribution of the events that are selected by the quality cuts. The results shown in the legend refer to the Gaussian fit of the distribution.

events, and provides full aperture at energies $E \gtrsim(6-7) \times 10^{19} \mathrm{eV}$. The observational duty cycle and the role of clouds are assessed. At highest energies, results indicate that the conversion factor between geometric aperture and exposure is at least 0.13 per year. The present results indicate that JEM-EUSO has the potential to reach an annual exposure of 9 times that of the Pierre Auger Observatory at energies around $10^{20} \mathrm{eV}$.

The methods currently developed to derive the arrival direction, energy and $X_{\max }$ of EAS are described in details and their current performance evaluated for limited classes of events. The results presented in this paper are compliant with the scientific requirements of the mission. Such methods are now under testing and optimization in order to provide their performance for a much wider class of events. These results will allow to evaluate the impact of the selection efficiency of events due to quality cuts on reconstruction to the science data analysis in the mission.

\section{Acknowledgments}

The majority of the content of this paper was presented at the COSPAR 2012 assembly in Mysore. This work is partially supported by Basic Science Interdisciplinary Research Projects of RIKEN and JSPS KAKENHI Grant (22340063, 23340081, and 24244042), by the Italian Ministry of Foreign Affairs, General Direction for the Cultural Promotion and Cooperation, by the 
Deutsches Zentrum für Luft- und Raumfahrt, by the 'Helmholtz Alliance for Astroparticle Physics HAP' funded by the Initiative and Networking Fund of the Helmholtz Association (Germany), and by Slovak Academy of Sciences MVTS JEM-EUSO as well as VEGA grant agency project 2/0076/13. The Spanish Consortium involved in the JEM-EUSO Space Mission is funded by MICINN under projects AYA2009-06037-E/ESP, AYA-ESP 2010-19082, AYA2011-29489-C03-01, AYA2012-39115-C03-01, CSD2009-00064 (Consolider MULTIDARK) and by Comunidad de Madrid (CAM) under project S2009/ ESP-1496. M.B. expresses his gratitude to the Japan Society for the Promotion of Science for providing him a Short-Term Invitation Fellowship for Research to accomplish part of the present studies at RIKEN.

The comments and criticisms of two anonymous referees which helped improving the quality of the paper are acknowledged.

\section{Appendix A. Auroral light and magnetospheric disturbance}

The position of auroral oval depends on the magnetospheric disturbance. With increasing level of the magnetospheric disturbance the equator-ward border of the auroral oval moves closer to the geomagnetic equator. The level of magnetospheric disturbance can be described by the Kp index. The equator-ward border position of auroral oval as a function of Kp index is usually fitted by linear functions (Whalen, 1985). Fits are evaluated for different magnetic local times with one hour steps. Table A.3 describes the position of equator-ward border geomagnetic latitude dependence on Kp index for midnight local magnetic time. Because at midnight the equator-ward auroral boundary position is one of the closest to the equator, we use these values as conservative estimation of possible appearance of aurorae. As the inclination of the ISS orbit is $51.6^{\circ}$ we cannot see aurorae during period with Kp index less then 3. The latitudinal thickness of the auroral oval is approximately $10^{\circ}$ in narrowest region for $\mathrm{Kp}=3$ and increases to $20^{\circ}$ for $\mathrm{Kp}=8$ (Milan et al., 2010). In conclusion we can assume that the ISS trajectory does not cross the pole-ward border of the auroral oval during the period 2000 till 2011.

To estimate the fraction of time in which auroral light hampers EAS measurements of JEM-EUSO experiment we evaluate the geomagnetic coordinates of the ISS real trajectory (every one-minute step taken from NASA SSC locator (NASA-SSCWEB)) together with adequate Kp index. We assume that auroral light is visible in JEM-EUSO nadir mode of observation if it is located inside auroral boundaries. In the model we exclude, for all 
Table A.3: Equator-ward auroral-boundary dependence between geomagnetic latitude and Kp index.

\begin{tabular}{c|c}
\hline Geomagnetic latitude $\left[^{\circ}\right]$ & Kp index \\
\hline 66.5 & 0 \\
64.5 & 1 \\
62.4 & 2 \\
60.4 & 3 \\
58.3 & 4 \\
56.3 & 5 \\
54.2 & 6 \\
52.2 & 7 \\
50.1 & 8 \\
48.1 & 9 \\
\hline
\end{tabular}

ISS positions, all the PDMs inside auroral boundaries on the northern and southern hemispheres from the time in which they could observe EAS.

The method is verified by the NASA video (NASA) taken on September 17, 2011 from 17:22 to 17:45 GMT, on an ascending node from south of Madagascar to north of Australia over the Indian Ocean. Part of the video shows the ISS flight over aurora Australis, when auroral light is visible in nadir observational mode. NASA video shows that the ISS passed over the Aurora approximately from 17:26 till 17:33 GMT. This is the time range predicted by our method. The positions of aurora visibility predicted by our method also approximately fit the positions of aurora visibility in the video as shown in Figure A.17.

Figure A.18 shows the parts of the ISS trajectory excluded from duty cycle for year 2003 when the magnetosphere was most disturbed and for year 2009 when situation was most quiet during the searched period. For year 2003 we exclude 12913 minutes, in 2009 only 608 minutes. Different levels of $\mathrm{Kp}$ indexes are indicated by different colors on excluded ISS positions. It is clear that with higher Kp indexes JEM-EUSO stays longer in regions where, during night time, aurorae can restrain measurements. But those periods are less frequent with increase of the Kp index as it can be seen on the histograms showing the distribution of Kp indexes during year 2003 and 2009 in the upper right corners of Figure A.18. We do not observe aurorae 


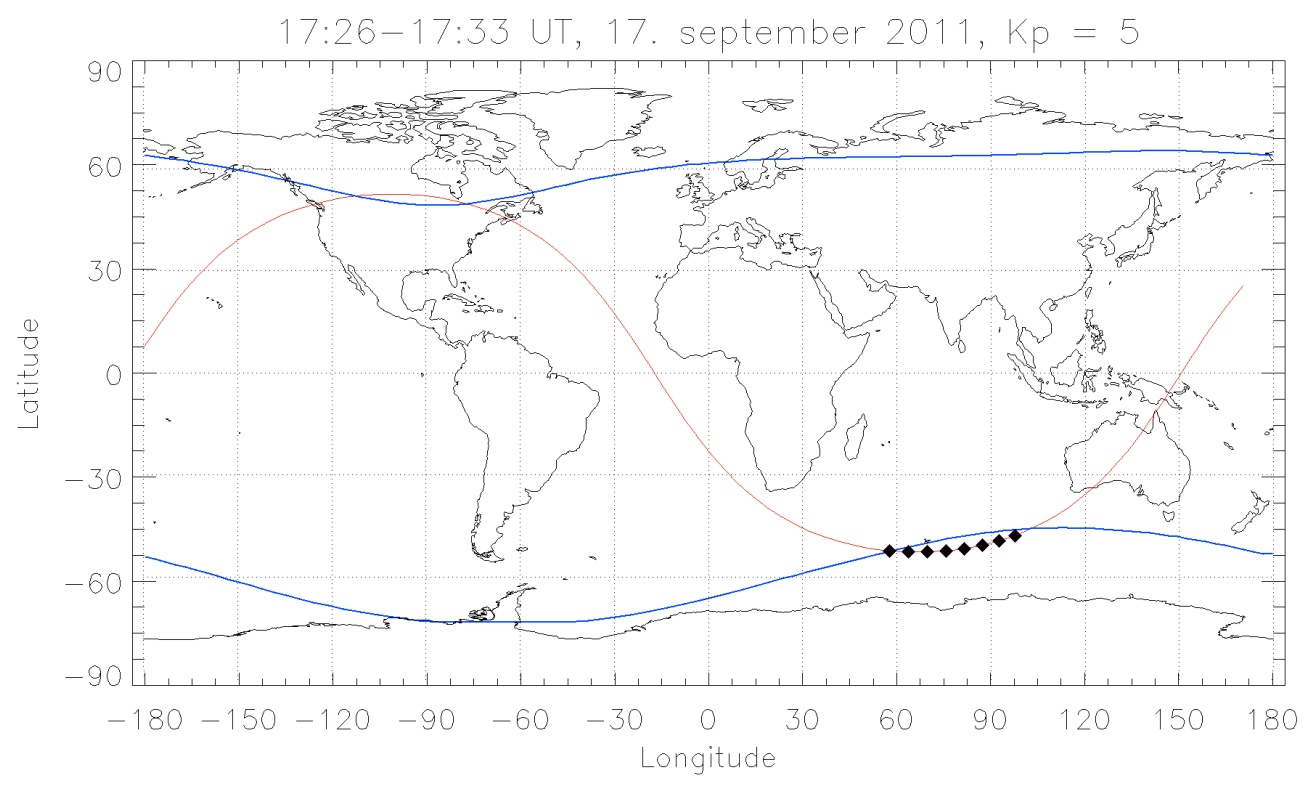

Figure A.17: Red line represents the trajectory of the ISS, blue lines are equator-ward boundaries of the auroral oval for magnetosphere disturbed at Kp index of 5. Diamonds on ISS trajectories are points on ISS trajectory with one-minute step from 17:26 till 17:33 GMT excluded from EAS measurements visible on NASA video (NASA).

inside equator-ward border along the ISS trajectory in nadir observation mode for Kp index equal two or lower values. While for most disturbed year (2003) we have $36 \%$ of time on orbit with $\mathrm{Kp}$ indexes of 0,1 or 2 , for most quiet year 2009 it is $94 \%$. Looking to periods where we can see aurorae more often, during 2003 we have $1.4 \%$ of time $\mathrm{Kp}$ indexes of 7,8 or 9 , while for year 2009 there are not disturbances at this levels at all. 

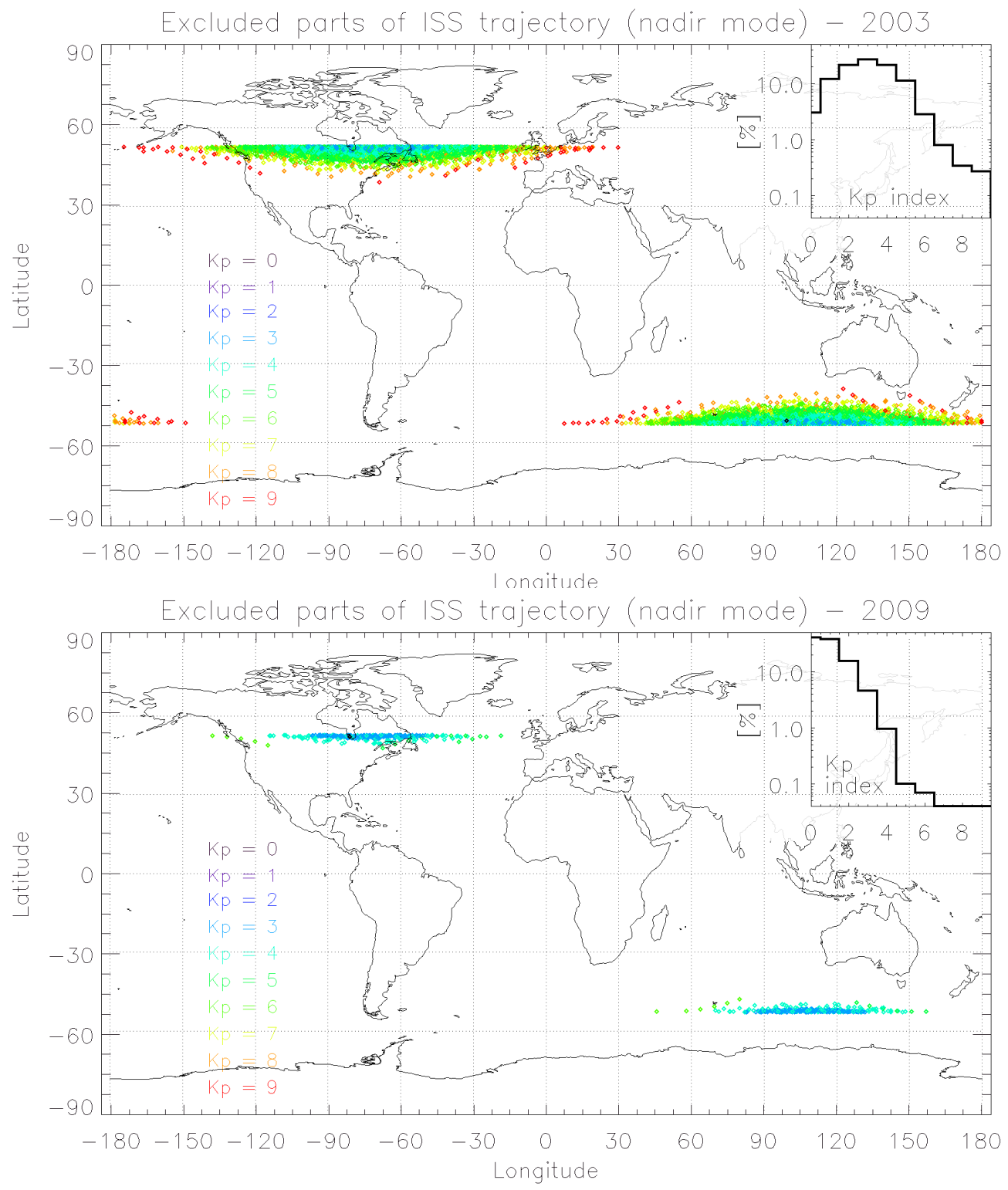

Figure A.18: Top and bottom plots show the excluded parts of the ISS trajectory due to aurorae during year 2003 and 2009, respectively. One-minute steps of trajectories are in color describing $\mathrm{Kp}$ index of geomagnetic activity. Inset figure displays the histogram of Kp index over each year. 


\section{References}

Abraham, J., Abreu, P., Aglietta, M., et al. (Auger Collaboration), Trigger and aperture of the surface detector array of the Pierre Auger Observatory, Nuclear Instruments and Methods A 613, 29-39, 2010.

Abu-Zayyad, T., Jui, C.C.H. and Loh, E.C., The effect of clouds on air showers observation from space, Astroparticle Physics 21, 163-182, 2004.

Adams Jr., J.H., Ahmad, S., Albert, J-N., et al. (JEM-EUSO Collaboration), An evaluation of the exposure in nadir observation of the JEM-EUSO mission, Astroparticle Physics 44, 76-90, 2013.

Agostinelli, S., Allison, J., Amako, E., et al., GEANT4 - a simulation toolkit, Nuclear Instruments and Methods A, 506, 250-303, 2003.

Berat, C., Bottai, S., De Marco, D., et al., Full simulation of space-based extensive air showers detectors with ESAF, Astroparticle Physics 33/4, 221-247, 2010.

Bergmann, T., Engel, R., Heck, D., et al., One-dimensional hybrid approach to extensive air shower simulation, Astroparticle Physics, 26, 420-432, 2007.

Bertaina, M., Bobik, P., Fenu, F., et al. (JEM-EUSO Coll), Requirements and expected performances of the JEM-EUSO mission, Proceedings of $32^{\text {nd }}$ International Cosmic Ray Conference, \# 0991, 2011;

Biktemerova, S., Gonchar, M., Naumov, D., et al. (JEM-EUSO Collaboration), Integration of ESAF and Geant4 for simulation of space based telescopes, Proceedings of $31^{\text {st }}$ International Cosmic Ray Conference, \# 1022, 2009.

Biktemerova, S.V., Gonchar, M.O., Geant4 tool for simulation of arbitrarily defined Fresnel lenses, Phys. Part. Nucl. Lett., 8, 789-793, 2011.

Bottai, S., Some algorithms for direction reconstruction in EUSO, EUSOSDA-REP-006-1, 2003

CACOLO: http://www.atmos.washington.edu/CloudMap/ 
Casolino, M., Adams, J.H., Bertaina, M.E., et al. (JEM-EUSO Collaboration), Detecting ultra-high energy cosmic rays from space with unprecedented acceptance: objectives and design of the JEM-EUSO mission, Astrophysics and Space Science Transactions 7, 477-482, 2011.

Catalano, O., Bertaina, M., Casolino, M., et al. (JEM-EUSO Collaboration), The trigger system of the JEM-EUSO Telescope, Proceedings of $31^{\text {st }}$ International Cosmic Ray Conference, 5, 1049-1052, 2009.

Ebisuzaki, T., Uehara, Y., Ohmori, H., et al. (JEM-EUSO Collaboration), The JEM-EUSO Project: Observing Extremely High Energy Cosmic Rays and Neutrinos from the International Space Station, Nuclear Physics B (Proceedings of Supplement) 175-176, 237-240, 2008.

Ebisuzaki, T., and JEM-EUSO Collaboration, The JEM-EUSO Mission, in this issue, 2013.

EUSO Collaboration, EUSO: Report on the Phase A Study, internal note EUSO-PI-REP-002, 2003.

Fenu, F., Mernik, T., Santangelo, A., et al. (JEM-EUSO Collaboration), The ESAF Simulation Framework for the JEM-EUSO mission, Proceedings of $32^{\text {nd }}$ International Cosmic Ray Conference, \# 0829, 2011; arXiv:1204.5065v1.

Fenu, F., Doctoral Thesis: A simulation study of the JEM-EUSO missions for Ultra High Energy Cosmic Ray Search, Eberhard Karls Universität Tübingen, 2013.

Garipov, G.K., Khrenov, B.A., Panasyuk, M.I., et al., UV radiation from the atmosphere: Results of the MSU "Tatiana" satellite measurements, Astroparticle Physics 24/4-5, 400-408, 2005.

Giller, M., Wieczorek, G., Kacperczyk, A., et al., Energy spectra of electrons in the extensive air showers of ultra-high energy, Journal of Physics G: Nuclear and Particle Physics, 30, 97-105, 2004.

Greisen, K., End to the Cosmic-Ray Spectrum?, Physical Review Letters 16, 748-750, 1966. 
Guzmán, A., Supanitsky, A., Iwotschkin, E., et al. (JEM-EUSO Collaboration), A study on JEM-EUSO's capability for neutrino-initiated EAS, Proceedings of $33^{\text {rd }}$ International Cosmic Ray Conference, ID0533, 2013; (preprint) arXiv:1307.7071.

Heck, D., Knapp, J., Capdevielle, J.N., et al., CORSIKA: A Monte Carlo Code to Simulate Extensive Air Showers, Forschungszentrum Karlsruhe Report FZKA6019, 1998.

Heinderickx, B, Quaghebeur, B., Speelman, E., et al., Spacecraft charging models in ESA's SPace ENVironment Information System (SPENVIS), Spacecraft Charging Technology, Proceedings of the Seventh International Conference, European Space Agency, ESA SP-476, 163-168, 2001.

Ilina, N.P., Kalmikov, N.N., Prosin, V.V., Cherenkov radiation and parameters of extensive air showers, Soviet Journal of Nuclear Physics, 55, 1540$1547,1992$.

ISCCP: http://isccp.giss.nasa.gov/

JEM-EUSO Collaboration, Purple Book 2010.

Kajino, F., Picozza, P., Ebisuzaki, T., et al. (JEM-EUSO Collaboration), Overview of the JEM-EUSO Instruments, Proceedings of $32^{\text {nd }}$ International Cosmic Ray Conference, 3, 282-285, 2011; (preprint) arXiv:1204.5065.

Kawasaki, Y., Bertaina, M.E., Sakaki, N., et al., Performance of a multianode photomultiplier employing a week electrostatic focusing system (Hamamatsu R8900 series), Nuclear Instruments and Methods A, 564/1, 378-394, 2006.

Kawasaki, Y., Casolino, M., Higashide, K., et al. (JEM-EUSO Collaboration), The Focal Surface of the JEM-EUSO instrument, Proceedings of $32^{\text {nd }}$ International Cosmic Ray Conference, \# 0472, 2011; arXiv:1204.5065v1.

Kneizys, F.X., Anderson, G.P., Shettle E.P. et al., User's Guide to LOWTRAN7, AFGL-TR-0177, U.S. Air Force Geophysics Laboratory, Hanscom AFB, Bedford, MA (1988). 
Mernik, T., Bobik, P., Putis, M., et al. (JEM-EUSO Collaboration), UV night background estimation in South Atlantic Anomaly, Proceedings of $33^{\text {rd }}$ International Cosmic Ray Conference, ID0874, 2013; (preprint) arXiv:1307.7071.

Milan, S. E., Evans, T. A., Hubert, B., Average auroral configuration parameterized by geomagnetic activity and solar wind conditions, Annales Geophysicae, 28, 10031012, 2010.

Montanet, F., Internal note of EUSO Collaboration, EUSO-SIM-REP-009$1.2,2004$.

Nagano, M., Kobayakawa, K., Sakaki, N., et al., New measurement on photon yields from air and the application to the energy estimation of primary cosmic rays, Astroparticle Physics, 22, 235-248, 2004.

NASA: http://www.nasa.gov/multimedia/videogallery/index.html?media_id=112491731

NASA-NOAA: http://www.ngdc.noaa.gov/dmsp/

NASA-SSCWEB: http://sscweb.gsfc.nasa.gov/cgi-bin/Locator.cgi

Neronov, A., Wada, S., Rodríguez Frías, M.D., et al. (JEM-EUSO Collaboration), Atmospheric Monitoring System of JEM-EUSO, Proceedings of $32^{\text {nd }}$ International Cosmic Ray Conference, 6, 332-335, 2011; (preprint) arXiv:1204.5065.

Neronov, A., Rodríguez Frías, M.D., Toscano, S., et al. (JEM-EUSO Collaboration), Atmospheric Monitoring System of the JEM-EUSO telescope, Proceedings of $33^{\text {nd }}$ International Cosmic Ray Conference, ID1072, 2013; (preprint) arXiv:1307.7071.

NOAA, NASA, US Air Force, "U.S. Standard Atmosphere 1976", U.S. Government Printing Office, Washington D.C., 1976.

Ostapchenko, S., Nonlinear screening effects in high energy hadronic interactions, Physical Review D 74, 014026/1-17, 2006.

Pallavicini, M., Pesce, R., Petrolini, A., et al., The observation of extensive air showers from an Earth-orbiting satellite, Astroparticle Physics 35/7, 402-420, 2012. 
Pesce, R., Master Thesis: Studio ed ottimizzazione delle prestazioni di un apparato per la rilevazione di raggi cosmici di altissima energia dallo spazio , University of Genova, 2003/2004, in Italian.

Rodríguez Frías, M.D., Morales de Los Ríos, J.A., del Peral, L., et al. (JEMEUSO Collaboration), The Atmospheric Monitoring System of the JEMEUSO space mission, EPJ Web of Conferences, 53, 10005/1-7, 2013.

Sáez-Cano, G., Shinozaki, K., del Peral, L., et al., Observation of extensive air showers in cloudy conditions by the JEM-EUSO Space Mission, to appear on Advances in Space Research, 2013; http://dx.doi.org/10.1016/j.asr.2013.07.015.

Schott: http://www.schott.com/advanced_optics/english/download/ schott_bandpass_bg3_2008_e.pdf

Shinozaki, K., Bertaina, M., Bobik, P., et al. (JEM-EUSO Collaboration), Overview of space-based ultra-high energy cosmic ray observation performance by JEM-EUSO mission, Proceedings of $33^{\text {rd }}$ International Cosmic Ray Conference, ID1250, 2013; (preprint) arXiv:1307.7071.

Strickland, D.J., Bishop, J., Evans, J.S, et al., Atmospheric Ultraviolet Radiance Integrated Code (AURIC): theory, software architecture, inputs, and selected results, Journal of Quantitative Spectroscopy and Radiative Transfer, 62/6, 689-742, 1999.

Taddei, E., Master Thesis: Studio di algoritmi per la ricostruzione della direzione degli sciami atmosferici in EUSO, University of Firenze, 2003/2004, in Italian.

Takahashi, Y., and JEM-EUSO Collaboration, The JEM-EUSO Mission, New Journal of Physics 11, 065009/1-21, 2009.

TOVS: http://www.ozonelayer.noaa.gov/action/tovs.htm/

Whalen, J.A., O’Neil, R.R., Picard, R.H., The Aurora, Chapter 12, Handbook of Geophysics and the Space Environment, Jursa, A.S, USAF, 1985.

Zatsepin, G.T. and Kuz'min, V.A., Upper Limit of the Spectrum of Cosmic Rays, Journal of Experimental and Theoretical Physics Letters 4, 78-80, 1966. 
Zuccaro Marchi, A., Takizawa, Y., Takahashi, Y., and JEM-EUSO Collaboration, The JEM-EUSO Optics Design, Proceedings of $32^{\text {nd }}$ International Cosmic Ray Conference, 3, 176-179, 2011; (preprint) arXiv:1204.5065. 